\title{
CONDITIONAL STABILITY ESTIMATES FOR ILL-POSED PDE PROBLEMS BY USING INTERPOLATION
}

\author{
U. TAUTENHAHN, U. HÄMARIK, B. HOFMANN, AND Y. SHAO
}

\begin{abstract}
The focus of this paper is on conditional stability estimates for illposed inverse problems in partial differential equations. Conditional stability estimates have been obtained in the literature by a couple different methods. In this paper we propose a method called interpolation method, which is based on interpolation in variable Hilbert scales. We are going to work out the theoretical background of this method and show that optimal conditional stability estimates are obtained. The capability of our method is illustrated by a comprehensive collection of different inverse and ill-posed PDE problems containing elliptic and parabolic problems, one source problem and the problem of analytic continuation.
\end{abstract}

\section{Contents}

1. Introduction 2

2. Conditional stability estimates by using interpolation 4

2.1. The interpolation method 4

2.2. Removing convexity 6

2.3. About the sharpness of the stability estimate $\quad 7$

3. Preliminaries on verifying convexity 11

3.1. Convexity for a first class of functions 11

3.2. Convexity for a second class of functions 13

4. Cauchy problems for elliptic equations 14

5. Cauchy problem for the Laplace equation in a strip 16

6. Cauchy problem for the Helmholtz equation 18

7. Backward heat conduction 21

8. Fractional backward heat conduction in a strip 24

9. Backward heat conduction in the plane 26

10. Non-standard sideways heat conduction 28

11. Fractional sideways heat conduction 30

12. Identification of heat sources 32

13. Analytic continuation 34

$\begin{array}{ll}\text { Acknowledgments } & 36\end{array}$

$\begin{array}{ll}\text { References } & 36\end{array}$

Date: July 25, 2011.

2000 Mathematics Subject Classification. 35R25, 35R30, 65J20, 65J22, 65M30, 65M32.

Key words and phrases. Ill-posed problems, inverse problems, conditional stability estimates, interpolation, elliptic problems, parabolic problems, source problems, analytic continuation. 


\section{Introduction}

Ill-posed problems for partial differential equations (PDE) arise in different mathematical models of natural sciences and engineering and have become increasingly important. In this paper we are interested in ill-posed PDE problems, mostly expressing linear inverse problems, that can be formulated in form of an operator equation

$$
A f=g,
$$

where we assume that $A: X \rightarrow Y$ is an injective and bounded linear operator mapping between infinite dimensional Hilbert spaces $X$ and $Y$ with inner products $(\cdot, \cdot)$ and norms $\|\cdot\|$. Ill-posedness means that the range $\mathcal{R}(A)$ of the operator $A$ is not closed, or in other words that $A^{-1}: \mathcal{R}(A) \subset Y \rightarrow X$ is an unbounded linear operator. This is equivalent to the fact that $\omega(\delta, X)=\infty$ for all $\delta>0$, where the quantity

$$
\omega(\delta, M)=\sup \{\|f\| \mid f \in M,\|A f\| \leq \delta\}
$$

denotes the modulus of continuity of the inverse operator $A^{-1}$ on the set $M \subseteq X$. As a consequence, in the ill-posed case there cannot exist continuous and nondecreasing finite functions $\beta(\delta), \delta>0$, with $\lim _{\delta \rightarrow 0} \beta(\delta)=0$, which we will call index functions, such that

$$
\|f\| \leq \beta(\|A f\|) \quad \text { for all } f \in X .
$$

However, when restricting the domain of the operator $A$ to certain subsets $M$ of $X$ and consequently the domain of $A^{-1}$ to images of $M$, the quantity $\omega(\delta, M)$ no longer needs to be infinite. If $M$ is bounded with $0 \in M$, then $\omega(\delta, M), \delta \geq 0$, is a bounded and non-decreasing function. This is also the case for unbounded $M$ when $M$ is a subset of a finite-dimensional subspace of $X$. For further properties of $\omega(\delta, M)$ we refer, e.g., to [32, 27, 29]. Most famously, Tikhonov's theorem (see $[63,64])$ asserts that $A^{-1}$ restricted to images of relatively compact sets $M$ is a continuous operator and as a consequence we have the limit condition

$$
\lim _{\delta \rightarrow 0} \omega(\delta, M)=0
$$

whenever $M$ is a convex set with $0 \in M$. Moreover, for such sets $M$ the modulus of continuity $\omega(\delta, M)$ is a continuous function for $0 \leq \delta<\infty$ and hence even an index function. As the following lemma will show in such case there exist index functions $\beta$ such that

$$
\|f\| \leq \beta(\|A f\|) \quad \text { for all } f \in M .
$$

Inequalities of the form (1.4) are conditional stability estimates on $M$ and we call index functions $\beta$ satisfying (1.4) conditional stability functions. The derivation of such functions $\beta$ for given compact subsets $M \subset X$ has attracted attention, see, e.g., $[1,67]$ and the reference cited therein. For its application in regularization, see, e.g., $[12,33,34,35]$.

Lemma 1.1. Let $M \subset X$ be such that $\omega(\delta, M)$ defined by (1.2) is finite and continuous for all $\delta>0$ and satisfies the limit condition (1.3). Then we have the following two properties: 
(i) The modulus of continuity $\omega$ is a conditional stability function on $M$, i.e.,

$$
\|f\| \leq \omega(\|A f\|, M) \quad \text { for all } f \in M .
$$

(ii) For any other index function $\beta$ that obeys (1.4) we have

$$
\omega(\|A f\|, M) \leq \beta(\|A f\|) \quad \text { for all } f \in M .
$$

Proof. The property (i) follows immediately from the definition (1.2) of $\omega$. On the other hand, the property (ii) is a consequence of the inequality chain

$$
\begin{gathered}
\omega(\|A f\|, M)=\sup \{\|\tilde{f}\| \mid \tilde{f} \in M,\|A \tilde{f}\| \leq\|A f\|\} \\
\leq \sup \{\beta(\|A \tilde{f}\|) \mid \tilde{f} \in M,\|A \tilde{f}\| \leq\|A f\|\} \leq \beta(\|A f\|),
\end{gathered}
$$

which makes use of the inequality (1.4) together with the fact that $\beta$ is a monotonically non-decreasing function.

As a consequence of the lemma all conditional stability functions $\beta$ on $M$ are majorant functions of the corresponding modulus of continuity. Vice versa, if $\omega(\delta, M)$ is an index function, then this is the smallest possible conditional stability function $\beta$ in (1.4).

There are different techniques for deriving conditional stability functions $\beta$ including the logarithmic convexity method, weighted energy methods, Gronwall's lemma and maximum principles [2, 30, 48], or methods based on Carleman estimates $[8,37,38,75]$. Let us explain by a simple model example the method of logarithmic convexity as one of the most traditional methods to obtain conditional stability functions $\beta$. This method is applicable to different kinds of ill-posed PDE problems.

Model example. Consider the heat equation problem backward in time, in which for fixed $t \in[0, T)$ the temperature $f:=u(\cdot, t)$ is to be determined from terminal data $g$ such that $u$ obeys the initial boundary value problem

$$
\left.\begin{array}{rll}
u_{t}-u_{x x}=0 & \text { for } & (x, t) \in(0, \pi) \times[0, T] \\
u(0, t)=u(\pi, t)=0 & \text { for } & t \in(0, T) \\
u(x, T)=g(x) & \text { for } & x \in(0, \pi)
\end{array}\right\} .
$$

The forward operator $A=A(t): L^{2}(0, \pi) \rightarrow L^{2}(0, \pi)$ mapping $f$ into $g$ is smoothing, and hence the problem $A f=g$ is ill-posed. For studying conditional stability, let us assume the solution smoothness $f \in M$ where

$$
M=\left\{u(\cdot, t) \in L^{2}(0, \pi) \mid u \text { obeys }(1.5),\|u(\cdot, 0)\|_{L^{2}(0, \pi)} \leq E\right\} .
$$

For this model example, the method of logarithmic convexity can be applied for deriving a conditional stability estimate on $M$ defined by (1.6). This method consists in executing the following working steps:

(a) We define the energy functional $F(t):=\|u(x, t)\|^{2}$.

(b) We show that $[\ln F(t)]^{\prime \prime}=\frac{F^{\prime \prime}(t) F(t)-\left[F^{\prime}(t)\right]^{2}}{F^{2}(t)} \geq 0$. 
(c) We conclude that $F(t) \leq F^{1-t / T}(0) F^{t / T}(T)$ which gives, for any fixed $t \in(0, T]$, the Hölder type conditional stability estimate

$$
\|u(x, t)\| \leq \beta(\|u(x, T)\|) \quad \text { with } \quad \beta(\delta)=E^{1-t / T} \delta^{t / T} .
$$

The main difficulty for obtaining an estimate (1.7) consists in proving the logarithmic convexity (b) of $F(t)$ taking into account that $F^{\prime}(t)=2\left(u, u_{t}\right)$ and $F^{\prime \prime}(t)=4\left(u_{t}, u_{t}\right)$. These identities imply by means of the Cauchy-Schwarz inequality the required non-negativity of $[\ln F(t)]^{\prime \prime}$.

\section{Conditional stability estimates by using interpolation}

2.1. The interpolation method. By interpolation in variable Hilbert scales we can estimate the intermediate norm $\|f\|$ if estimates for some weaker norm $\|A f\|$ and some stronger norm $\left\|\left[\varphi\left(A^{*} A\right)\right]^{-1 / 2} f\right\|$ are known. Variable Hilbert scale inequalities have been introduced by Hegland, see [24, 25]. Such inequalities which extend the classical interpolation inequality became a powerful tool in the analysis of regularization under general smoothness conditions, see, e.g., [7, 26, 28, 40, 41, $42,45,46,47,56,61]$. Variable Hilbert scale interpolation is sometimes also called interpolation with a function parameter, see $[6,44]$. In this paper we use a variant of the variable Hilbert scale approach based on Jensen's inequality for deriving sharp conditional stability estimates on special subsets $M \subset X$, which have the structure

$$
M=M_{\varphi, E}=\left\{f \in X \mid f=\left[\varphi\left(A^{*} A\right)\right]^{1 / 2} v,\|v\| \leq E\right\},
$$

i.e., the admissible elements $f \in X$ satisfy a general source condition generated by some index function $\varphi$.

Theorem 2.1. Assume that $f \in M_{\varphi, E}$, where $M_{\varphi, E}$ is given by (2.1) for some index function $\varphi:(0, a] \rightarrow(0, \varphi(a)]$ with $a=\left\|A^{*} A\right\|$. Assume further that the function $\varrho(\lambda):=\lambda \varphi^{-1}(\lambda), \varrho:(0, \varphi(a)] \rightarrow(0, a \varphi(a)]$ is convex. Then we have

$$
\|f\| \leq \beta(\|A f\|) \quad \text { with } \quad \beta(\delta)=E \sqrt{\varrho^{-1}\left(\delta^{2} / E^{2}\right)} \text {. }
$$

Proof. We follow the ideas outlined in [61, Theorem 2.1]. Let $E_{\lambda}$ the spectral family of $A^{*} A$. Since $\varrho$ is convex we may employ Jensen's inequality and obtain due to $\varrho(\varphi(\lambda))[\varphi(\lambda)]^{-1}=\lambda$ that

$$
\varrho\left(\frac{\|f\|^{2}}{\left\|\left[\varphi\left(A^{*} A\right)\right]^{-1 / 2} f\right\|^{2}}\right) \leq \frac{\int \varrho(\varphi(\lambda))[\varphi(\lambda)]^{-1} \mathrm{~d}\left\|E_{\lambda} f\right\|^{2}}{\left\|\left[\varphi\left(A^{*} A\right)\right]^{-1 / 2} f\right\|^{2}}=\frac{\|A f\|^{2}}{\left\|\left[\varphi\left(A^{*} A\right)\right]^{-1 / 2} f\right\|^{2}},
$$

or equivalently,

$$
\left\|\left[\varphi\left(A^{*} A\right)\right]^{-1 / 2} f\right\|^{2} \varrho\left(\frac{\|f\|^{2}}{\left\|\left[\varphi\left(A^{*} A\right)\right]^{-1 / 2} f\right\|^{2}}\right) \leq\|A f\|^{2} .
$$

Since $\varphi$ is an index function, $\varphi^{-1}(t):=t^{-1} \varrho(t)$ is increasing. Hence, $t \rightarrow t \varrho(1 / t)$ is decreasing. Consequently, since $\left\|\left[\varphi\left(A^{*} A\right)\right]^{-1 / 2} f\right\| \leq E$, the above estimate gives

$$
E^{2} \varrho\left(\|f\|^{2} / E^{2}\right) \leq\|A f\|^{2} \text {. }
$$

By rearranging terms this yields $\|f\| \leq \beta(\|A f\|)$ with $\beta(\delta)=E \sqrt{\varrho^{-1}\left(\delta^{2} / E^{2}\right)}$. 
Remark 2.2. Since the index function $\varphi$ in Theorem 2.1 is defined on $(0, a]$ it follows that the index function $\rho(\lambda):=\lambda \varphi^{-1}(\lambda)$, which could also implicitly be defined by $\varrho(\varphi(\lambda)):=\lambda \varphi(\lambda)$, is defined on $(0, \varphi(a)]$. The range of $\varrho$ is $(0, a \varphi(a)]$. Consequently, $\varrho^{-1}:(0, a \varphi(a)] \rightarrow(0, \varphi(a)]$. Hence, for the stability function $\beta$ we have $\beta:(0, c] \rightarrow(0, d]$ with $c=E \sqrt{a \varphi(a)}$ and $d=E \sqrt{\varphi(a)}$. Since for $f \in M_{\varphi, E}$ we have $\|A f\|^{2}=\left(A^{*} A \varphi\left(A^{*} A\right) v, v\right) \leq E^{2} a \varphi(a)$, there follows that $\|A f\|$ belongs to the domain of $\beta$ for all elements $f \in M_{\varphi, E}$.

Let us give some comment on Theorem 2.1. In many inverse partial differential equation problems this theorem makes it possible to derive explicit or implicit formulae for conditional stability functions $\beta$ on special sets $M$ that arise by imposing a bound on a part of the solution of the partial differential equation. In the next sections we apply Theorem 2.1 for obtaining conditional stability estimates for a collection of different ill-posed PDE problems. Our way can be summarized as follows:

Interpolation method for deriving conditional stability estimates. Assuming the solution smoothness $f \in M \subset X$, execute the following four steps:

(i) Reformulate the ill-posed PDE problem as an operator equation (1.1).

(ii) Derive the function $\varphi$ such that the set $M$ coincides with the set $M_{\varphi, E}$ given by (2.1). Verify that $\varphi$ is an index function.

(iii) Derive the function $\varrho(\lambda):=\lambda \varphi^{-1}(\lambda)$ and verify its convexity.

(iv) Derive a formula for $\beta$ as given in (2.2).

Let us illustrate these working steps by the model example discussed in the introduction. For the working step (i) we use the method of separation of variables and obtain the operator equation

$$
A f=g \quad \text { with } \quad A(t) f(t)=\sum_{i=1}^{\infty}\left(f(t), u_{i}\right) e^{-i^{2}(T-t)} u_{i},
$$

where $u_{i}=\sqrt{2 / \pi} \sin i x$. That is, $A(t)$ is an self-adjoint operator with eigenvalues $e^{-i^{2}(T-t)}$ and eigenelements $u_{i}$. For the working step (ii) one shows that the set $M$ defined by (1.6) has the equivalent form

$$
M=\left\{u(\cdot, t) \in L^{2}(0, \pi) \mid u(\cdot, t)=A(t)^{p} v,\|v\| \leq E, p=\frac{t}{T-t}\right\},
$$

that is, the set $M$ coincides with the set $M_{\varphi, E}$ given by (2.1) where $\varphi$ is given by

$$
\varphi(\lambda)=\lambda^{t /(T-t)}, \quad \varphi:\left(0, e^{-2(T-t)}\right] \rightarrow\left(0, e^{-2 t}\right] .
$$

In the working step (iii) we verify $\varrho(\lambda):=\lambda \varphi^{-1}(\lambda)$ as $\varrho(\lambda)=\lambda^{T / t}$, which is always convex. Then we can find in the final working step (iv) according to (2.2) the required conditional stability function as

$$
\beta(\delta)=E^{1-t / T} \delta^{t / T} .
$$

This function coincides with the conditional stability function obtained by the method of logarithmic convexity as outlined in the introduction. 
2.2. Removing convexity. Theorem 2.1 requires convexity of the function $\varrho$, which for special problems is sometimes difficult to check or even violated. Our next theorem does not require this convexity assumption. The proof is based on cross-connections between the best possible worst case error on $M$ and the modulus of continuity of the inverse $A^{-1}$ on $M$ and requires following notations:

(a) Let $\mathcal{R}: Y \rightarrow X$ be an arbitrary operator and $\mathcal{R}\left(g^{\delta}\right)$ be an approximate solution for the solution $f^{\dagger}$ of equation (1.1) from noisy data $g^{\delta}$ that obey $\left\|g-g^{\delta}\right\| \leq \delta$. Then the quantity

$$
\Delta(\delta, \mathcal{R})=\sup \left\{\left\|\mathcal{R}\left(g^{\delta}\right)-f\right\| \mid\left\|A f-g^{\delta}\right\| \leq \delta, f \in M\right\}
$$

is called worst case error of the method $\mathcal{R}$ on the set $M$. This quantity characterizes the accuracy of the method $\mathcal{R}$ in the worst case sense.

(b) An optimal method $\mathcal{R}_{\text {opt }}$ is characterized by

$$
\Delta\left(\delta, \mathcal{R}_{\text {opt }}\right)=\inf _{\mathcal{R}} \Delta(\delta, \mathcal{R}) \quad\left(0<\delta \leq \delta_{0}\right)
$$

and this quantity is called best possible worst case error on the set $M$.

Theorem 2.3. Assume that $f \in M_{\varphi, E}$ where $M_{\varphi, E}$ is given by (2.1) for some index function $\varphi:(0, a] \rightarrow(0, \varphi(a)]$ with $a=\left\|A^{*} A\right\|$, and let $\varrho(\lambda):=\lambda \varphi^{-1}(\lambda)$. Then we have

$$
\|f\| \leq \beta(\|A f\|) \quad \text { with } \quad \beta(\delta)=\sqrt{2} E \sqrt{\varrho^{-1}\left(\delta^{2} / E^{2}\right)} .
$$

Proof. In analogy to Vainikkos result [65, Lemma 2.2] which is based on a minimax property given in [43] we obtain for linear methods $R_{\alpha}: Y \rightarrow X$ that the worst case error on $M_{\varphi, E}$ obeys

$$
\Delta^{2}\left(\delta, R_{\alpha}\right)=\inf _{0<\xi<1}\left\|\frac{E^{2}}{\xi}\left(I-R_{\alpha} A\right) \varphi\left(A^{*} A\right)\left(I-R_{\alpha} A\right)^{*}+\frac{\delta^{2}}{1-\xi} R_{\alpha} R_{\alpha}^{*}\right\| .
$$

For standard regularization methods $R_{\alpha} g^{\delta}=g_{\alpha}\left(A^{*} A\right) A^{*} g^{\delta}$ we obtain (compare also [61, formula (4.6)]) that

$$
\Delta^{2}\left(\delta, R_{\alpha}\right)=\inf _{0<\xi<1} \sup _{0<\lambda \leq a}\left(\frac{E^{2}}{\xi}\left(1-\lambda g_{\alpha}(\lambda)\right)^{2} \varphi(\lambda)+\frac{\delta^{2}}{1-\xi} \lambda g_{\alpha}^{2}(\lambda)\right) .
$$

For methods that obey $0 \leq 1-\lambda g_{\alpha}(\lambda) \leq 1$ we choose $\xi=1-\lambda g_{\alpha}(\lambda)$ and obtain

$$
\Delta^{2}\left(\delta, R_{\alpha}\right) \leq \sup _{0<\lambda \leq a}\left(E^{2}\left(1-\lambda g_{\alpha}(\lambda)\right) \varphi(\lambda)+\delta^{2} g_{\alpha}(\lambda)\right) .
$$

Choosing for $g_{\alpha}$ the spectral method with $g_{\alpha}(\lambda)=1 / \lambda$ for $\lambda \geq \alpha$ and $g_{\alpha}(\lambda)=1 / \alpha$ for $\lambda<\alpha$ we obtain that the best possible worst case error on $M_{\varphi, E}$ obeys

$$
\Delta^{2}\left(\delta, \mathcal{R}_{\mathrm{opt}}\right) \leq \inf _{\alpha>0} \sup _{t \in[0,1]}\left(E^{2}(1-t) \varphi(\alpha t)+\delta^{2} / \alpha\right) .
$$

We use the estimate $\sup _{t \in[0,1]}(1-t) \varphi(\alpha t) \leq \varphi(\alpha)$ and obtain

$$
\Delta^{2}\left(\delta, \mathcal{R}_{\mathrm{opt}}\right) \leq \inf _{\alpha>0}\left(E^{2} \varphi(\alpha)+\delta^{2} / \alpha\right)
$$


For further estimating we use a special $\alpha$ for which both summands on the righthand side of (2.8) are equal, that is, we choose $\alpha$ as the unique solution of the equation $\alpha \varphi(\alpha)=\delta^{2} / E^{2}$, or equivalently $\varrho(\varphi(\alpha))=\delta^{2} / E^{2}$, and obtain

$$
\Delta^{2}\left(\delta, \mathcal{R}_{\text {opt }}\right) \leq 2 E^{2} \varrho^{-1}\left(\delta^{2} / E^{2}\right) .
$$

Furthermore, from $[32,66]$ we know that for arbitrary centrally symmetric and convex sets $M \subset X$ there holds the estimate $\omega(\delta, M) \leq \Delta\left(\delta, \mathcal{R}_{\text {opt }}\right)$. From this estimate and (2.9) we obtain that

$$
\omega^{2}\left(\delta, M_{\varphi, E}\right) \leq 2 E^{2} \varrho^{-1}\left(\delta^{2} / E^{2}\right)
$$

for all $\delta:=\|A f\|, f \in M_{\varphi, E}$. Since $\omega$ is a conditional stability function, that is, there holds $\|f\| \leq \omega\left(\|A f\|, M_{\varphi, E}\right)$, we obtain the result of the theorem.

Remark 2.4. We should note that in the special case of polynomial index functions $\varphi(\lambda)=\lambda^{p}$ in (2.1), the inf-sup problem (2.7) can be solved analytically. Solving the inner sup problem shows that the sup is attained at $t_{0}=\frac{p}{p+1}$. The remaining outer inf problem is then to minimize $g(\alpha):=E^{2} p^{p} \alpha^{p} /(p+1)^{p+1}+\delta^{2} / \alpha$. The inf is attained at $\alpha_{0}=\frac{p+1}{p}\left(\frac{\delta}{E}\right)^{\frac{2}{p+1}}$ and leads to the estimate

$$
\Delta^{2}\left(\delta, \mathcal{R}_{\mathrm{opt}}\right) \leq E^{\frac{2}{p+1}} \delta^{\frac{2 p}{p+1}}=E^{2} \varrho^{-1}\left(\delta^{2} / E^{2}\right) .
$$

2.3. About the sharpness of the stability estimate. Our next theorem tells us that the stability estimate of Theorem 2.1 is sharp, that is, there exist elements $f \in M_{\varphi, E}$ for which we have equality in (2.2). This result does not require the convexity assumption for the function $\varrho(\lambda):=\lambda \varphi^{-1}(\lambda)$.

Theorem 2.5. Let $M_{\varphi, E}$ be given by (2.1) for some index function $\varphi:(0, a] \rightarrow$ $(0, \varphi(a)]$ with $a=\left\|A^{*} A\right\|$, let $\varrho(\lambda):=\lambda \varphi^{-1}(\lambda)$ and assume that $\delta^{2} / E^{2} \in \sigma(H)$ where $\sigma(H)$ denotes the spectrum of $H=A^{*} A \varphi\left(A^{*} A\right)$. Then,

$$
\omega\left(\delta, M_{\varphi, E}\right) \geq \beta(\delta) \quad \text { with } \quad \beta(\delta)=E \sqrt{\varrho^{-1}\left(\delta^{2} / E^{2}\right)} .
$$

Proof. The first part of the proof is borrowed from [61, proof of Theorem 2.1]. For $M=M_{\varphi, E}$, the function $\omega$ defined by (1.2) may be rewritten as

$$
\omega\left(\delta, M_{\varphi, E}\right)=\sup \left\{\left\|\left[\varphi\left(A^{*} A\right)\right]^{1 / 2} v\right\| \mid\|v\| \leq E,\left\|A\left[\varphi\left(A^{*} A\right)\right]^{1 / 2} v\right\| \leq \delta\right\} .
$$

Let $\delta^{2} / E^{2}$ be an eigenvalue of the operator $H=A^{*} A \varphi\left(A^{*} A\right)$ and $v_{0}$ the corresponding eigenelement with $\left\|v_{0}\right\|=E$. Then we have

$$
A^{*} A \varphi\left(A^{*} A\right) v_{0}=\left(\delta^{2} / E^{2}\right) v_{0} .
$$

Consequently, $\left\|A\left[\varphi\left(A^{*} A\right)\right]^{1 / 2} v_{0}\right\|^{2}=\left(\delta^{2} / E^{2}\right)\left(v_{0}, v_{0}\right)=\delta^{2}$. That is, the element $v_{0}$ obeys the side conditions in the function $\omega\left(\delta, M_{\varphi, E}\right)$. Hence,

$$
\omega^{2}\left(\delta, M_{\varphi, E}\right) \geq\left\|\left[\varphi\left(A^{*} A\right)\right]^{1 / 2} v_{0}\right\|^{2}=\left(\varphi\left(A^{*} A\right) v_{0}, v_{0}\right) .
$$

From $\varrho(\varphi(\lambda))=\lambda \varphi(\lambda)$ we have $\varrho\left(\varphi\left(A^{*} A\right)\right) v_{0}=H v_{0}=\left(\delta^{2} / E^{2}\right) v_{0}$. Consequently, $\varphi\left(A^{*} A\right) v_{0}=\varrho^{-1}\left(\delta^{2} / E^{2}\right) v_{0}$, which gives $\omega^{2}\left(\delta, M_{\varphi, E}\right) \geq E^{2} \varrho^{-1}\left(\delta^{2} / E^{2}\right)$. From this estimate we obtain (2.11) in case that $\delta^{2} / E^{2}$ is an eigenvalue of the operator $H$. 
In the second part we treat the case if $\delta^{2} / E^{2}$ belongs to the limit spectrum $\sigma_{l}(H)$ of the operator $H$, which is the spectrum of $H$ excluding eigenvalues. From Weyl [68], see also [58, Section 133], we have the equivalence result

$$
\lambda_{0} \in \sigma_{l}\left(A^{*} A\right) \Leftrightarrow\left\{\exists \text { sequence }\left(v_{n}\right) \mid\left\|v_{n}\right\|=1, v_{n} \rightarrow 0, A^{*} A v_{n}-\lambda_{0} v_{n} \rightarrow 0\right\} .
$$

Clearly, in Weyl's result, the norm condition $\left\|v_{n}\right\|=1$ may be replaced by the norm condition $\left\|v_{n}\right\|=E$ with arbitrary $E>0$, since also $E v_{n}$ converges weakly to 0 and $A^{*} A\left(E v_{n}\right)-\lambda_{0}\left(E v_{n}\right) \rightarrow 0$. Let $\psi$ be an arbitrary index function. We use that $\psi\left(A^{*} A\right)=\int_{0}^{a} \psi(\lambda) \mathrm{d} E_{\lambda}$ and obtain that Weyl's result attains the form

$$
\psi\left(\lambda_{0}\right) \in \sigma_{l}\left(\psi\left(A^{*} A\right)\right) \Leftrightarrow\left\{\exists\left(v_{n}\right) \mid\left\|v_{n}\right\|=E, v_{n} \rightarrow 0, \psi\left(A^{*} A\right) v_{n}-\psi\left(\lambda_{0}\right) v_{n} \rightarrow 0\right\} .
$$

Now we assume that $\delta^{2} / E^{2} \in \sigma_{l}(H)$ where $H=A^{*} A \varphi\left(A^{*} A\right)$. It follows that $\psi\left(\delta^{2} / E^{2}\right) \in \sigma_{l}(\psi(H))$. We use the notation $\varrho^{-1 / 2}(t):=\sqrt{\varrho^{-1}(t)}$ where $\varrho^{-1}$ is the inverse of $\varrho$, apply the above equivalence result with $\psi(\lambda)=\lambda^{1 / 2}$ and $\psi(\lambda)=$ $\varphi^{1 / 2}(\lambda)=\varrho^{-1 / 2}(\lambda \varphi(\lambda))$, respectively, and obtain that there exists some sequence $\left(v_{n}\right)$ with $\left\|v_{n}\right\|=E$ such that

$$
\begin{aligned}
& \text { (I) }\left\|H^{1 / 2} v_{n}-(\delta / E) v_{n}\right\| \rightarrow 0, \\
& \text { (II) }\left\|\left[\varphi\left(A^{*} A\right)\right]^{1 / 2} v_{n}-\varrho^{-1 / 2}\left(\delta^{2} / E^{2}\right) v_{n}\right\| \rightarrow 0 .
\end{aligned}
$$

We note that for different index functions $\psi$ one may use the same sequence $\left(v_{n}\right)$ as for the function $\psi(\lambda)=\lambda$. This can easily be checked for power functions $\psi(\lambda)=\lambda^{p}$, therefore it also holds for polynomials, and consequently for any index functions since index functions can be approximated by polynomials. From (I) and (II) we conclude that for arbitrary $\varepsilon>0$ there is an index $n=n(\varepsilon)$ such that

$$
\begin{array}{ll}
(\mathrm{I})^{\prime} & \left\|H^{1 / 2} v_{n}\right\| \leq(\delta / E)\left\|v_{n}\right\|+\varepsilon=\delta+\varepsilon, \\
(\mathrm{II})^{\prime} & \left\|\left[\varphi\left(A^{*} A\right)\right]^{1 / 2} v_{n}\right\| \geq \varrho^{-1 / 2}\left(\delta^{2} / E^{2}\right)\left\|v_{n}\right\|-\varepsilon=E \varrho^{-1 / 2}\left(\delta^{2} / E^{2}\right)-\varepsilon .
\end{array}
$$

From both estimates $(\mathrm{I})^{\prime}$, (II) $)^{\prime}$ and the equality $\left\|H^{1 / 2} v_{n}\right\|=\left\|A\left[\varphi\left(A^{*} A\right)\right]^{1 / 2} v_{n}\right\|$ we conclude that

$$
\begin{aligned}
\omega\left(\delta+\varepsilon, M_{\varphi, E}\right) & =\sup \left\{\left\|\left[\varphi\left(A^{*} A\right)\right]^{1 / 2} v\right\|\|\| v\|\leq E,\| A\left[\varphi\left(A^{*} A\right)\right]^{1 / 2} v \| \leq \delta+\varepsilon\right\} \\
& \geq E \varrho^{-1 / 2}\left(\delta^{2} / E^{2}\right)-\varepsilon .
\end{aligned}
$$

Since $\varepsilon>0$ is arbitrary, we obtain $\omega\left(\delta, M_{\varphi, E}\right) \geq E \varrho^{-1 / 2}\left(\delta^{2} / E^{2}\right)$.

Remark 2.6. For compact operators $A^{*} A$ with eigenvalues $\lambda_{i}$ and corresponding eigenelements $u_{i}, i=1,2, \ldots$, the proof of the first part of Theorem 2.5 shows following: There exists a sequence $\left(f_{i}\right)$, given by $\left(f_{i}\right)=\left(E \sqrt{\varphi\left(\lambda_{i}\right)} u_{i} /\left\|u_{i}\right\|\right)$, with properties $f_{i} \in M_{\varphi, E},\left\|A f_{i}\right\| \rightarrow 0$ for $i \rightarrow \infty$ and

$$
\left\|f_{i}\right\|^{2}=E^{2} \varrho^{-1}\left(\left\|A f_{i}\right\|^{2} / E^{2}\right)
$$

that is, the conditional stability estimate given by (2.2) is sharp.

Our next proposition tells us that in special situations the modulus of continuity is smaller than $E \sqrt{\varrho^{-1}\left(\delta^{2} / E^{2}\right)}$. However, such special situations cannot occur if $A^{*} A$ has pure continuous spectrum. 
Proposition 2.7. Let $\varphi$ be an index function, let $\varrho(\lambda):=\lambda \varphi^{-1}(\lambda)$ be convex, let $\psi(\lambda):=\varrho(\varphi(\lambda))=\lambda \varphi(\lambda)$, let $A$ be compact and let $\lambda_{1}>\lambda_{2}>\ldots$ be the distinct eigenvalues of $A^{*} A$. Then,

$$
\omega\left(\delta, M_{\varphi, E}\right)=E \sqrt{s\left(\delta^{2} / E^{2}\right)} \quad \text { for } \quad 0<\delta^{2} / E^{2} \leq \psi\left(\lambda_{1}\right)
$$

where $s$ is the linear spline that interpolates the points $\left(\psi\left(\lambda_{i}\right), \varphi\left(\lambda_{i}\right)\right), i=1,2, \ldots$ If $\delta^{2} / E^{2} \in\left(\psi\left(\lambda_{i+1}\right), \psi\left(\lambda_{i}\right)\right)$ and $\varrho$ is stricly convex on this interval, then

$$
\omega\left(\delta, M_{\varphi, E}\right)<E \sqrt{\varrho^{-1}\left(\delta^{2} / E^{2}\right)} .
$$

Proof. The statement (2.12) can be concluded from [31], see also [41, Theorem 1]. For $\delta^{2} / E^{2} \in\left(\psi\left(\lambda_{i+1}\right), \psi\left(\lambda_{i}\right)\right)$ there exists $t \in(0,1)$ such that

$$
\delta^{2} / E^{2}=t \psi\left(\lambda_{i}\right)+(1-t) \psi\left(\lambda_{i+1}\right) .
$$

From (2.12) we conclude that for these $\delta$-values we have

$$
\omega\left(\delta, M_{\varphi, E}\right)=E \sqrt{t \varphi\left(\lambda_{i}\right)+(1-t) \varphi\left(\lambda_{i+1}\right)} .
$$

From the strict convexity of $\varrho$ we have

$$
\begin{aligned}
\varrho\left(t \varphi\left(\lambda_{i}\right)+(1-t) \varphi\left(\lambda_{i+1}\right)\right) & <t \varrho\left(\varphi\left(\lambda_{i}\right)\right)+(1-t) \varrho\left(\varphi\left(\lambda_{i+1}\right)\right) \\
& =t \psi\left(\lambda_{i}\right)+(1-t) \psi\left(\lambda_{i+1}\right)=\delta^{2} / E^{2} .
\end{aligned}
$$

Now (2.13) follows from (2.14) and (2.15).

Remark 2.8. To summarize, concerning upper and lower bounds of $\omega$ we know:

(i) If $\varrho$ is convex, then we have from Theorem 2.1 and Theorem 2.5

$\left(\mathrm{i}_{1}\right) \omega\left(\delta, M_{\varphi, E}\right)=E \sqrt{\varrho^{-1}\left(\delta^{2} / E^{2}\right)}$ for $\delta^{2} / E^{2} \in \sigma(H)$ with $H=A^{*} A \varphi\left(A^{*} A\right)$.

$\left(\mathrm{i}_{2}\right) \omega\left(\delta, M_{\varphi, E}\right) \leq E \sqrt{\varrho^{-1}\left(\delta^{2} / E^{2}\right)}$ for $\delta^{2} / E^{2} \notin \sigma(H)$ where, due to Proposition 2.7 , for strictly convex functions $\varrho$ and compact operators $A^{*} A$ the left-hand side is properly smaller.

(ii) If $\varrho$ is not necessarily convex, then by Theorems 2.3 and 2.5 we have

(ii $\left.{ }_{1}\right) E \sqrt{\varrho^{-1}\left(\delta^{2} / E^{2}\right)} \leq \omega\left(\delta, M_{\varphi, E}\right) \leq \sqrt{2} E \sqrt{\varrho^{-1}\left(\delta^{2} / E^{2}\right)}$ for $\delta^{2} / E^{2} \in \sigma(H)$, and we do not know sharper bounds of $\omega$.

$\left(\mathrm{ii}_{2}\right) \omega\left(\delta, M_{\varphi, E}\right) \leq \sqrt{2} E \sqrt{\varrho^{-1}\left(\delta^{2} / E^{2}\right)}$ for $\delta^{2} / E^{2} \notin \sigma(H)$.

From part $\left(\mathrm{ii}_{1}\right)$ of Remark 2.8 we conclude that for $\delta \rightarrow 0$ the rate $\omega^{2}\left(\delta, M_{\varphi, E}\right)=$ $o\left(\varrho^{-1}\left(\delta^{2} / E^{2}\right)\right)$ cannot hold. From parts $\left(\mathrm{i}_{2}\right)$ and $\left(\mathrm{ii}_{2}\right)$ of Remark 2.8 there arises the question concerning lower bounds for $\omega\left(\delta, M_{\varphi, E}\right)$ in the case $\delta^{2} / E^{2} \notin \sigma(H)$. In the paper [41, Corollary 1], some lower bound is given provided $\varrho$ is convex and certain weak assumptions on the decay rate of the eigenvalues $\lambda_{i}$ of the operator $A^{*} A$ and on the index function $\varphi$ are valid. Our next proposition shows that some lower bounds for $\omega\left(\delta, M_{\varphi, E}\right)$ hold also true in situations where $\varrho$ is not necessarily convex. We divide the $\delta$-interval into subintervals

$$
\delta_{1}>\delta_{2}>\ldots>\delta_{i}>\delta_{i+1}>\ldots \quad \text { with } \delta_{i}^{2}=E^{2} \lambda_{i} \varphi\left(\lambda_{i}\right)
$$

and obtain following lower bounds for $\omega\left(\delta, M_{\varphi, E}\right)$ :

Proposition 2.9. Let $\varphi$ be an index function, let $\varrho(\lambda):=\lambda \varphi^{-1}(\lambda)$, let $A^{*} A$ be compact and let $\lambda_{1}>\lambda_{2}>\ldots$ be the ordered distinct eigenvalues of $A^{*} A$, then: 
(i) The modulus of continuity obeys the estimate

$$
\omega^{2}\left(\delta, M_{\varphi, E}\right) \geq E^{2} \frac{\left(\delta^{2}-\delta_{i+1}^{2}\right) \varphi\left(\lambda_{i}\right)+\left(\delta_{i}^{2}-\delta^{2}\right) \varphi\left(\lambda_{i+1}\right)}{\delta_{i}^{2}-\delta_{i+1}^{2}} \quad \text { for } \quad \delta \in\left[\delta_{i+1}, \delta_{i}\right] .
$$

(ii) Let $c_{i}$ be positive constants with $\varphi\left(\lambda_{i+1}\right) \geq c_{i} \varphi\left(\lambda_{i}\right)$, then

$$
\omega^{2}\left(\delta, M_{\varphi, E}\right) \geq c_{i} E^{2} \varrho^{-1}\left(\delta^{2} / E^{2}\right) \quad \text { for } \quad \delta \in\left[\delta_{i+1}, \delta_{i}\right] .
$$

(iii) If there exists some constant $k>0$ such that $c_{i} \geq k$ for all $i \in \mathbb{N}$, then

$$
\omega^{2}\left(\delta, M_{\varphi, E}\right) \geq k E^{2} \varrho^{-1}\left(\delta^{2} / E^{2}\right) \quad \text { for } \quad \delta \in\left(0, \delta_{1}\right] .
$$

Proof. For the eigenvalues $\lambda_{i}$ of the operator $A^{*} A$, let $u_{i}$ the corresponding eigenelements with length $\left\|u_{i}\right\|=E$. We construct the element $v$ by

$$
v=\alpha u_{i}+\beta u_{i+1} \quad \text { with } \quad \alpha^{2}=\frac{\delta^{2}-\delta_{i+1}^{2}}{\delta_{i}^{2}-\delta_{i+1}^{2}} \quad \text { and } \quad \beta^{2}=\frac{\delta_{i}^{2}-\delta^{2}}{\delta_{i}^{2}-\delta_{i+1}^{2}} .
$$

For this element $v$ we have

$$
\|v\|^{2}=\alpha^{2} E^{2}+\beta^{2} E^{2}=E^{2} \quad \text { and } \quad\left\|A\left[\varphi\left(A^{*} A\right)\right]^{1 / 2} v\right\|^{2}=\delta_{i}^{2} \alpha^{2}+\delta_{i+1}^{2} \beta^{2}=\delta^{2} .
$$

Hence, this element $v$ obeys the both side conditions of the function

$$
\omega^{2}\left(\delta, M_{\varphi, E}\right)=\sup \left\{\left\|\left[\varphi\left(A^{*} A\right)\right]^{1 / 2} v\right\|^{2} \mid\|v\|^{2} \leq E^{2},\left\|A\left[\varphi\left(A^{*} A\right)\right]^{1 / 2} v\right\|^{2} \leq \delta^{2}\right\} .
$$

Consequently,

$$
\omega^{2}\left(\delta, M_{\varphi, E}\right) \geq\left\|\left[\varphi\left(A^{*} A\right)\right]^{1 / 2} v\right\|^{2}=E^{2} \alpha^{2} \varphi\left(\lambda_{i}\right)+E^{2} \beta^{2} \varphi\left(\lambda_{i+1}\right),
$$

and the proof of estimate (2.16) is complete. Next, let us prove (2.17). Since $\varphi$ is monotonically increasing we have $\varphi\left(\lambda_{i}\right) \geq \varphi\left(\lambda_{i+1}\right)$. Consequently,

$$
\omega^{2}\left(\delta, M_{\varphi, E}\right) \geq E^{2}\left(\alpha^{2}+\beta^{2}\right) \varphi\left(\lambda_{i+1}\right)=E^{2} \varphi\left(\lambda_{i+1}\right) .
$$

We use that $\varphi\left(\lambda_{i+1}\right) \geq c_{i} \varphi\left(\lambda_{i}\right)$, take into account that $\varrho^{-1}\left(\delta_{i}^{2} / E^{2}\right)=\varrho^{-1}\left(\lambda_{i} \varphi\left(\lambda_{i}\right)\right)=$ $\varphi\left(\lambda_{i}\right)$, use that $\varrho^{-1}$ is increasing and obtain

$$
\omega^{2}\left(\delta, M_{\varphi, E}\right) \geq E^{2} c_{i} \varphi\left(\lambda_{i}\right)=E^{2} c_{i} \varrho^{-1}\left(\delta_{i}^{2} / E^{2}\right) \geq E^{2} c_{i} \varrho^{-1}\left(\delta^{2} / E^{2}\right) .
$$

The proof of part (iii) follows from part (ii).

Remark 2.10. The assumption in part (iii) of the proposition is satisfied if the eigenvalues $\lambda_{i}$ of the operator $A^{*} A$ tend to zero not too fast. Let us discuss two examples where $\varphi$ is given by $\varphi(\lambda)=\lambda^{p}$ with $p>0$, then:

(i) In case $\lambda_{i}=1 / i^{\nu}$ with some constant $\nu>0$, for the constants $c_{i}$ we have $c_{i}=\left(\frac{i}{i+1}\right)^{\nu p} \geq\left(\frac{1}{2}\right)^{\nu p}$. Hence, the assumption in part (iii) of Proposition 2.9 is satisfied with $k=2^{-\nu p}$.

(ii) In case $\lambda_{i}=\exp \left(-i^{\nu}\right)$, for the constants $c_{i}$ we have $c_{i}=\exp \left(p i^{\nu}-p(i+1)^{\nu}\right)$. Hence, the assumption in part (iii) of Proposition 2.9 is satisfied for $\nu \leq 1$, but violated for $\nu>1$.

Some sufficient conditions which guarantee that the assumption in part (iii) of Proposition 2.9 is satisfied are that

(i) there exists some constant $\gamma>0$ with $\lambda_{i+1} / \lambda_{i} \geq \gamma(i=1,2, \ldots)$ and

(ii) $\varphi$ satisfies a $\Delta_{2}$-condition, that is, there holds $\varphi(2 t) \leq c_{2} \varphi(t)$ for $t \in\left(0, \frac{a}{2}\right]$. 
The $\Delta_{2}$-condition has been introduced in [41] and implies the existence of some $c_{\gamma}>0$ such that $\varphi(\gamma t) \geq c_{\gamma} \varphi(t)$. Hence, $\varphi\left(\lambda_{i}\right)=\varphi\left(\frac{\lambda_{i+1}}{\lambda_{i}} \lambda_{i}\right) \geq \varphi\left(\gamma \lambda_{i}\right) \geq c_{\gamma} \varphi\left(\lambda_{i}\right)$, which yields the assumption from part (iii) of Proposition 2.9 with $k=c_{\gamma}$.

\section{Preliminaries on verifying convexity}

3.1. Convexity for a first class of functions. We consider the following function $\varrho(\lambda), \lambda \in\left(0, \lambda\left(\xi_{0}\right)\right]$, which is given in parameter representation by

$$
\left.\begin{array}{l}
\lambda(\xi)=c_{1}\left[\frac{\cosh (y \xi)}{\left(c_{2}+\xi^{2}\right)^{p / 2} \cosh \xi}\right]^{2} \\
\varrho(\xi)=c_{1}\left[\frac{1}{\left(c_{2}+\xi^{2}\right)^{p / 2} \cosh \xi}\right]^{2}
\end{array}\right\} \quad\left(\xi_{0} \leq \xi<\infty\right) .
$$

The functions $\varrho$ of the forthcoming Sections 4 - 6 can all be rewritten into the equivalent form (3.1) with constants

$$
c_{1}>0, \quad c_{2} \geq 0, \quad \xi_{0} \geq 0, \quad y \in(0,1] \quad \text { and } \quad p \geq 0 .
$$

Our aim consists in finding necessary and sufficient conditions under which the function $\varrho(\lambda), \lambda \in\left(0, \lambda\left(\xi_{0}\right)\right]$, is convex. In our study we use the standard notations $\varrho^{\prime}=\frac{\mathrm{d}}{\mathrm{d} \lambda} \varrho(\lambda), \dot{\lambda}=\frac{\mathrm{d}}{\mathrm{d} \xi} \lambda(\xi), \dot{\varrho}=\frac{\mathrm{d}}{\mathrm{d} \xi} \varrho(\xi)$. In addition, our study is based on following two auxiliary functions

$$
r(\xi):=\cosh ^{2}(y \xi) \text { and } h(\xi):=\frac{2 p \xi}{c_{2}+\xi^{2}}+2 \tanh \xi .
$$

Proposition 3.1. The function $\varrho(\lambda)$, defined in parameter representation by (3.1), is convex if and only if

$$
h^{2}+\dot{h} \geq h \ddot{r} / \dot{r} \quad \text { for all } \xi \in\left[\xi_{0}, \infty\right) .
$$

Proof. Since $\xi \rightarrow \tanh \xi$ is monotonically increasing it can be verified that

$$
\dot{\lambda}=-2 \lambda(\xi)\left[\frac{p \xi}{c_{2}+\xi^{2}}+\tanh \xi-y \tanh (y \xi)\right]<0 .
$$

Consequently, since $\varrho^{\prime \prime}=(\ddot{\varrho} \dot{\lambda}-\dot{\varrho} \ddot{\lambda}) / \dot{\lambda}^{3}$ we obtain that $\varrho^{\prime \prime} \geq 0$ is equivalent to $\ddot{\varrho} \dot{\lambda} \leq \dot{\varrho} \ddot{\lambda}$. We note that $\lambda(\xi)=\varrho(\xi) r(\xi)$ with $r$ given by (3.2). Hence, from $\dot{\lambda}=\dot{\varrho} r+\varrho \dot{r}$ and $\ddot{\lambda}=\ddot{\varrho} r+2 \dot{\varrho} \dot{r}+\varrho \ddot{r}$ we obtain that $\ddot{\varrho} \dot{\lambda} \leq \dot{\varrho} \ddot{\lambda}$ is equivalent to $\dot{r}\left[\varrho \ddot{\varrho}-2 \dot{\varrho}^{2}\right] \leq \varrho \ddot{\varrho} \ddot{r}$. Since $\dot{r}>0$, this inequality is equivalent to

$$
\varrho \varrho \varrho-2 \dot{\varrho}^{2} \leq \varrho \ddot{\varrho} \ddot{r} / \dot{r} \text { for all } \xi \in\left[\xi_{0}, \infty\right) \text {. }
$$

We compute $\dot{\varrho}$ and obtain $\dot{\varrho}(\xi)=-\varrho(\xi) h(\xi)<0$ with $h$ given by (3.2). For $\ddot{\varrho}$ we

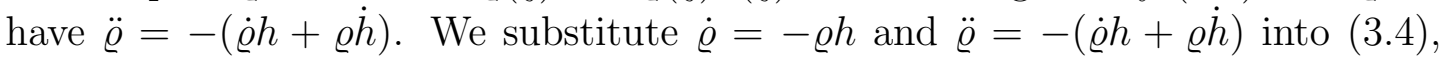
collect terms and obtain (3.3).

Proposition 3.2. The function $\varrho(\lambda)$, defined in parameter representation by (3.1), is convex if and only if

$$
p^{2}+\psi_{1}(\xi, y) \cdot p+\psi_{2}(\xi, y) \geq 0 \quad \text { for all } \xi \in\left[\xi_{0}, \infty\right)
$$


where $\psi_{1}$ and $\psi_{2}$ are given by

$$
\begin{aligned}
& \psi_{1}(\xi, y)=\frac{c_{2}+\xi^{2}}{\xi}[2 \tanh \xi-y \operatorname{coth}(2 y \xi)]+\frac{c_{2}-\xi^{2}}{2 \xi^{2}} \\
& \psi_{2}(\xi, y)=\frac{\left(c_{2}+\xi^{2}\right)^{2}}{\xi^{2}} \tanh \xi[\operatorname{coth}(2 \xi)-y \operatorname{coth}(2 y \xi)]
\end{aligned}
$$

Proof. For $\dot{h}$ we have $\dot{h}=2 p\left(c_{2}-\xi^{2}\right) /\left(c_{2}+\xi^{2}\right)^{2}+2-2 \tanh ^{2} \xi$. We substitute $h$ and $\dot{h}$ into (3.3), rearrange terms and obtain

$$
\begin{aligned}
p^{2} \cdot \frac{2 \xi^{2}}{\left(c_{2}+\xi^{2}\right)^{2}} & +p \cdot\left[\frac{4 \xi}{c_{2}+\xi^{2}} \tanh \xi+\frac{c_{2}-\xi^{2}}{\left(c_{2}+\xi^{2}\right)^{2}}\right]+1+\tanh ^{2} \xi \\
& \geq\left[p \cdot \frac{\xi}{c_{2}+\xi^{2}}+\tanh \xi\right] \cdot \frac{\ddot{r}}{\dot{r}}
\end{aligned}
$$

From $\dot{r}=y \sinh (2 y \xi)$ and $\ddot{r}=2 y^{2} \cosh (2 y \xi)$ we have $\ddot{r} / \dot{r}=2 y \operatorname{coth}(2 y \xi)$. We substitute this into (3.8), rearrange terms, use in addition that $1+\tanh ^{2} \xi=$ $2 \tanh \xi \operatorname{coth}(2 \xi)$ and obtain the statement of the proposition.

Let us discuss some monotonicity properties of the functions $\psi_{1}(\xi, y)$ and $\psi_{2}(\xi, y)$ :

(a) The function $y \rightarrow y \operatorname{coth} y$ is monotonically increasing. It follows that for any fixed $\xi>0$, both $\psi_{1}(\xi, y)$ and $\psi_{2}(\xi, y)$ are monotonically decreasing with respect to $y \in(0,1]$.

(b) For any fixed $y \in(0,1]$, both $\psi_{1}(\xi, y)$ and $\psi_{2}(\xi, y)$ are monotonically increasing with respect to $\xi \in(0, \infty)$.

From these properties and Proposition 3.2 we have

Corollary 3.3. The function $\varrho(\lambda)$, defined in parameter representation by (3.1), is convex if and only if

$$
p^{2}+\psi_{1}\left(\xi_{0}, y\right) p+\psi_{2}\left(\xi_{0}, y\right) \geq 0 .
$$

This convexity condition is valid

(i) for all $y \in(0,1]$ if and only if $p^{2}+p \cdot \psi_{1}\left(\xi_{0}, 1\right) \geq 0$,

(ii) for all $p \geq 0$ if and only if $\psi_{1}\left(\xi_{0}, y\right)+2 \sqrt{\psi_{2}\left(\xi_{0}, y\right)} \geq 0$,

(iii) for all $p \geq 0$ and all $y \in(0,1]$ if and only if $\psi_{1}\left(\xi_{0}, 1\right) \geq 0$.

Proof. The proof of (3.9) follows from Proposition 3.2 and the monotonicity property (b). The proof of part (i) follows from part (3.9), the monotonicity property (a) and $\psi_{2}\left(\xi_{0}, 1\right)=0$. For the proof of (ii) we consider (3.9) and distinguish two cases $\psi_{1}\left(\xi_{0}, y\right) \geq 0$ and $\psi_{2}\left(\xi_{0}, y\right)<0$. In the first case, convexity of $\varrho$ is guaranteed for all $p \geq 0$ (since $\psi_{2} \geq 0$ ). In the second case, convexity of $\varrho$ is guaranteed for all $p \geq 0$ if and only if $\psi_{1}^{2} \leq 4 \psi_{2}$, or equivalently, $\psi_{1}+2 \sqrt{\psi_{2}} \geq 0$. From both cases we obtain (ii). The final part (iii) follows from part (i).

Since $\dot{\lambda}(\xi)<0$, the domain $\lambda \in\left(0, \lambda\left(\xi_{0}\right)\right]$ of the function $\varrho(\lambda)$ is largest for $\xi_{0}=0$. In this case we conclude from Corollary 3.3 the following 
Corollary 3.4. Let $\xi_{0}=0$. Then, the function $\varrho(\lambda)$ defined in parameter representation by (3.1) is convex if and only if

$$
p^{2}+p\left[2 c_{2}\left(1-y^{2} / 3\right)-1\right]+2 c_{2}^{2}\left(1-y^{2}\right) / 3 \geq 0 .
$$

This inequality is valid

(i) for all $y \in(0,1]$ if and only if $p^{2}+p\left[4 c_{2} / 3-1\right] \geq 0$,

(ii) for all $p \geq 0$ if and only if $c_{2}\left[1-y^{2} / 3+\sqrt{2\left(1-y^{2}\right) / 3}\right] \geq 1 / 2$,

(iii) for all $p \geq 0$ and all $y \in(0,1]$ if and only if $c_{2} \geq 3 / 4$.

Proof. The convexity condition (3.10) follows from (3.9) and the limit relations

$$
\lim _{\xi \rightarrow 0} \psi_{1}(\xi, y)=2 c_{2}\left(1-y^{2} / 3\right)-1 \text { and } \lim _{\xi \rightarrow 0} \psi_{2}(\xi, y)=2 c_{2}^{2}\left(1-y^{2}\right) / 3 .
$$

From (3.10) we obtain (i), (ii) and (iii).

3.2. Convexity for a second class of functions. We consider the following function $\varrho(\lambda), \lambda \in\left(0, \lambda\left(\xi_{0}\right)\right]$, which is given in parameter representation by

$$
\left.\begin{array}{l}
\lambda(\xi)=c_{1}\left(c_{2}+\xi^{c_{3}}\right)^{-p} \mathrm{e}^{-z \xi} \\
\varrho(\xi)=c_{1}\left(c_{2}+\xi^{c_{3}}\right)^{-p} \mathrm{e}^{-\xi}
\end{array}\right\} \quad\left(\xi_{0} \leq \xi<\infty\right) .
$$

The functions $\varrho$ of the forthcoming Sections 7, 8, 9, 11, 13 can all be rewritten into the equivalent form (3.11) with constants

$$
c_{1}>0, \quad c_{2} \geq 0, \quad c_{3} \geq 1, \quad \xi_{0} \geq 0, \quad z \in[0,1) \quad \text { and } \quad p \geq 0 .
$$

Our aim consists in finding necessary and sufficient conditions under which the function $\varrho(\lambda), \lambda \in\left(0, \lambda\left(\xi_{0}\right)\right]$, is convex.

Proposition 3.5. The function $\varrho(\lambda)$, defined in parameter representation by (3.11), is convex if and only if

$$
p^{2} \psi^{2}(\xi)+p[(z+1) \psi(\xi)+\dot{\psi}(\xi)]+z \geq 0 \text { for all } \xi \in\left[\xi_{0}, \infty\right)
$$

where $\psi(\xi)$ is given by

$$
\psi(\xi)=\frac{c_{3} \xi^{c_{3}-1}}{c_{2}+\xi^{c_{3}}}
$$

Proof. Computing $\dot{\lambda}$ yields $\dot{\lambda}=-\lambda(\xi)[p \psi(\xi)+z]<0$. We note that the argument $\lambda=\lambda(\xi)$ possesses the representation $\lambda(\xi)=\varrho(\xi) r(\xi)$ with $r$ given by

$$
r(\xi):=\mathrm{e}^{(1-z) \xi} .
$$

We proceed as in the proof of Proposition 3.1 and obtain that $\varrho(\lambda), \lambda \in\left(0, \lambda\left(\xi_{0}\right)\right]$, is convex if and only if

$$
\varrho \varrho \varrho-2 \dot{\varrho}^{2} \leq \varrho \ddot{\varrho} \ddot{r} / \dot{r} \quad \text { for all } \xi \in\left[\xi_{0}, \infty\right) .
$$

Computing $\dot{\varrho}$ and $\ddot{\varrho}$ yields $\dot{\varrho}=-(p \psi+1) \varrho<0$ and $\ddot{\varrho}=-(p \psi+1) \dot{\varrho}-p \dot{\psi} \varrho$. We substitute this into (3.14), observe that $\ddot{r} / \dot{r}=1-z$ and obtain

$$
(p \psi+1)^{2}+p \dot{\psi} \geq(p \psi+1)(1-z) .
$$

Rearranging terms yields (3.12). 
From (3.12) we conclude that $\varrho(\lambda)$ is convex for all $z \in[0,1)$ in the cases $p=0$ and $p \geq 1$. The second result follows from (3.12) and $\dot{\psi}=\psi\left[\left(c_{3}-1\right) / \xi-\psi\right] \geq$ $-\psi^{2}$. Hence, non-convexity cannot hold for smoothness situations with $p=0$ and $p \geq 1$. From Proposition 3.5 we have

Corollary 3.6. The function $\varrho(\lambda)$, defined in parameter representation by (3.11), is convex for all $z \in[0,1)$ and all $p \geq 0$ if and only if

$$
c_{2} \geq \sup _{\xi \in\left[\xi_{0}, \infty\right)} g(\xi) \quad \text { with } \quad g(\xi)=\frac{\xi^{c_{3}}(1-\xi)}{\xi+c_{3}-1} .
$$

Proof. The left hand side of (3.12) is monotonically increasing with respect to $z \in[0,1)$. It follows that the convexity condition (3.12) holds true for all $z \in[0,1)$

if and only if $p^{2} \psi^{2}(\xi)+p[\psi(\xi)+\dot{\psi}(\xi)] \geq 0$ for all $\xi \in\left[\xi_{0}, \infty\right)$. From this we conclude that $\varrho(\lambda)$ is convex for all $z \in[0,1)$ and all $p \geq 0$ if and only if

$$
\psi(\xi)+\dot{\psi}(\xi) \geq 0 \text { for all } \xi \in\left[\xi_{0}, \infty\right) .
$$

Using that $\dot{\psi}=\psi\left[\left(c_{3}-1\right) / \xi-\psi\right]$ we obtain by rearranging terms that this convexity condition is valid if and only if (3.15) holds.

\section{Cauchy problems for elliptic equations}

This problem is taken from $[49,53,59]$. Problems of this kind are ill-posed and arise in several fields of physics and engineering such as hydrodynamics, tomography, theory of electronic signals, non-destructive testing, geophysics, seismology and others.

System equation formulation of the problem: Determine, for any fixed $y \in(0,1]$, the function $f(x):=u(x, y), x \in \Omega \subset \mathbb{R}^{n}$, from the data function $g(x):=u(x, 0)$ where $u(x, y)$ obeys the following Cauchy problem

$$
\left.\begin{array}{rlrl}
-L u+u_{y y} & =0 & & \text { for } \quad x \in \Omega \subset \mathbb{R}^{n}, 0<y<1 \\
u(x, 0) & =g(x) & & \text { for } \quad x \in \Omega \subset \mathbb{R}^{n} \\
u_{y}(x, 0) & =0 & & \text { for } \quad x \in \Omega \subset \mathbb{R}^{n}
\end{array}\right\}
$$

and $L: D(L) \subset X \rightarrow X$ denotes a linear densely defined self adjoint and positive definite operator in $X=L^{2}(\Omega)$ with eigenvalues

$$
1=l_{1} \leq l_{2} \leq \ldots \leq l_{i} \leq \ldots, \quad l_{i} \rightarrow \infty \quad \text { for } i \rightarrow \infty
$$

and eigenelements $\left\{u_{i}\right\}$ that form an orthonormal basis in $X$.

Special case: An example for (4.1) is the Laplace equation in two dimensions

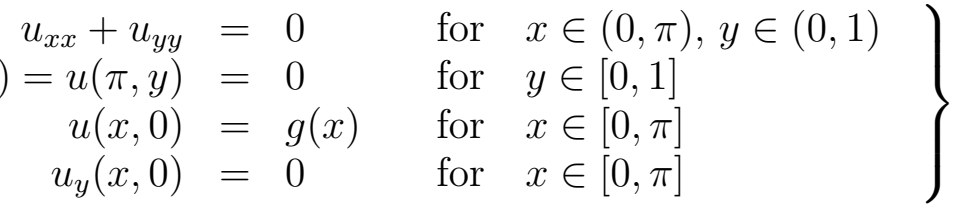

in which the eigenvalues $l_{i}$ and eigenelements $u_{i}$ of $L: H_{0}^{1}(0, \pi) \cap H^{2}(0, \pi) \subset$ $X \rightarrow X$ with $X=L^{2}(0, \pi)$ are given by $l_{i}=i^{2}, u_{i}=\sqrt{\frac{2}{\pi}} \sin (i x)(i=1,2, \ldots)$. Under the smoothness assumption $\|u(x, 1)\| \leq E$, this example has been treated 
in [35] by using the method of logarithmic convexity. We will treat more general smoothness. However, we do not know if our more general solution smoothness could also be treated by the method of logarithmic convexity.

Smoothness assumption: For studying conditional stability, we assume the solution smoothness $\left\|L^{p / 2} u(x, 1)\right\| \leq E$ with some $E>0$ and $p \geq 0$ in case $y \in(0,1)$ and $p>0$ in case $y=1$, that is,

$$
u(\cdot, y) \in M=\left\{u(\cdot, y) \in X \mid u \text { obeys }(4.1),\left\|L^{p / 2} u(\cdot, 1)\right\| \leq E\right\},
$$

and ask for a stability estimate $\|f\| \leq \beta(\|g\|)$.

Step 1 (Operator equation formulation of the problem): By the method of separation of variables we have for problem (4.1) the unique solution

$$
u(x, y)=\sum_{i=1}^{\infty}\left(u(x, 0), u_{i}\right) \cosh \left(\sqrt{l_{i}} y\right) u_{i},
$$

consequently, $\left(u(x, 0), u_{i}\right)=\left(u(x, y), u_{i}\right) / \cosh \left(\sqrt{l_{i}} y\right)$, which shows us that the operator $A=A(y): X \rightarrow X$ of the operator equation $A f=g$ has the representation

$$
A(y) u(x, y)=\sum_{i=1}^{\infty} \frac{\left(u(x, y), u_{i}\right)}{\cosh \left(\sqrt{l_{i}} y\right)} u_{i}
$$

We realize that $A(y): X \rightarrow X$ is a linear self-adjoint compact operator with eigenvalues $s_{i}=1 / \cosh \left(\sqrt{l_{i}} y\right)$ and eigenelements $u_{i}$. Since the eigenvalues $s_{i}$ of the operator $A(y)$ decay exponentially fast we realize that the problem is a severely ill-posed problem. The ill-posedness becomes worse as $y$ increases.

Step 2 (Deriving the index function $\varphi$ ): Now we ask the question if the set $M$ from (4.3) is equivalent to some general source set

$$
M_{\varphi, E}=\left\{u(\cdot, y) \in X \mid u(\cdot, y)=\left[\varphi\left(A^{*} A\right)\right]^{1 / 2} v,\|v\| \leq E\right\}
$$

with some index function $\varphi=\varphi(\lambda)$. Some formal computations show that both sets (4.3) and (4.4) are equal for $\varphi:\left(0,1 / \cosh ^{2} y\right] \rightarrow\left(0, \cosh ^{2} y / \cosh ^{2} 1\right]$ implicitly given by

$$
\varphi\left(1 / \cosh ^{2}(\sqrt{l} y)\right)=l^{-p} \cosh ^{2}(\sqrt{l} y) / \cosh ^{2}(\sqrt{l}), \quad 1 \leq l<\infty .
$$

In analogy to [59, Proposition 3.2] it can be shown that the function $\varphi$ defined by (4.5) is an index function.

Step 3 (Deriving the function $\varrho$ and verifying its convexity): From (4.5) we obtain that the function $\varrho:\left(0, \cosh ^{2} y / \cosh ^{2} 1\right] \rightarrow\left(0,1 / \cosh ^{2} 1\right]$ is implicitly given by

$$
\varrho\left(l^{-p} \cosh ^{2}(\sqrt{l} y) / \cosh ^{2}(\sqrt{l})\right)=l^{-p} / \cosh ^{2}(\sqrt{l}), \quad 1 \leq l<\infty .
$$

We substitute in (4.6) $\sqrt{l}=\xi$ and see that $\varrho$ defined by (4.6) has the special form (3.1) with constants

$$
c_{1}=1, \quad c_{2}=0 \quad \text { and } \quad \xi_{0}=1 .
$$

From Corollary 3.3 we have 
Proposition 4.1. Let $\psi_{1}(\xi, y)$ and $\psi_{2}(\xi, y)$ be defined by (3.6), (3.7) with constant $c_{2}=0$. Then, the function $\varrho(\lambda)$, defined in parameter representation by (3.1) with constants (4.7), is convex if and only if $(y, p) \in(0,1] \times[0, \infty)$ obey

$$
p^{2}+\psi_{1}(1, y) p+\psi_{2}(1, y) \geq 0 \text {. }
$$

This inequality is valid

(i) for all $y \in(0,1]$ if and only if $p=0$ or $p \geq-\psi_{1}(1,1) \approx 0.0141264$,

(ii) for all $p \geq 0$ if and only if $y \leq y^{*}$ with $y^{*} \approx 0.999926$.

Step 4 (Deriving the conditional stability estimate): Due to the preparatory steps $1-3$ we are now able to apply Theorem 2.1 and Theorem 2.3 and obtain

Theorem 4.2. Let the convexity assumption (4.8) hold. Then, on the set $M$ given by (4.3) we have for any fixed $y \in(0,1)$ an improved Hölder type conditional stability estimate and for $y=1$ some logarithmic type conditional stability estimate $\|f\| \leq \beta(\|g\|)$ with

$$
\beta(\delta)=E l_{0}^{-p / 2} \cosh \sqrt{l_{0}} y / \cosh \sqrt{l_{0}}
$$

where $l_{0}$ is the unique solution of the equation $l^{p / 2} \cosh \sqrt{l}=E / \delta$. For $\delta \rightarrow 0$ there holds the asymptotic representation

$$
\beta(\delta)=E^{y}\left(\frac{\delta}{2}\right)^{1-y}\left(\ln \frac{1}{\delta}\right)^{-p y}(1+o(1)) .
$$

If the convexity assumption (4.8) is violated, then (4.9) and (4.10) hold true with an additional factor $\sqrt{2}$ on the right hand side.

Proof (sketch). From (4.6) we obtain that $\varrho^{-1}$ is implicitly given by

$$
\varrho^{-1}\left(l^{-p} / \cosh ^{2}(\sqrt{l})\right)=l^{-p} \cosh ^{2}(\sqrt{l} y) / \cosh ^{2}(\sqrt{l}), \quad 1 \leq l<\infty .
$$

The proof of (4.9) follows from (4.11) and Theorem 2.1. Further, from (4.11) we obtain that $\varrho^{-1}$ possesses the asymptotic representation

$$
\varrho^{-1}(\lambda)=(\lambda / 4)^{1-y}(-\ln \sqrt{\lambda})^{-2 p y}(1+o(1)) \quad \text { for } \quad \lambda \rightarrow 0 .
$$

From this representation and Theorem 2.1 we obtain (4.10).

\section{Cauchy problem for the Laplace equation in a strip}

Problems of this type have been under consideration, e.g., in the papers [11, $20,39,53]$.

System equation formulation of the problem: Determine, for any fixed $y \in(0,1]$, the function $f(x):=u(x, y)$ from the data function $g(x):=u(x, 0)$ where $u(x, y)$ obeys the following Cauchy problem for the two-dimensional Laplace equation in the strip $0<y \leq 1$ :

$$
\left.\begin{array}{rlrl}
u_{x x}+u_{y y} & =0 & & \text { for } \quad x \in \mathbb{R}, 0<y \leq 1 \\
u(x, 0) & =g(x) & & \text { for } \quad x \in \mathbb{R} \\
u_{y}(x, 0) & =0 & & \text { for } \quad x \in \mathbb{R}
\end{array}\right\}
$$


Smoothness assumption: Let $X=L^{2}(\mathbb{R})$ with norm $\|\cdot\|$. For studying conditional stability, we assume for some $E>0$ and $p \geq 0$ in case $y \in(0,1)$ and $p>0$ in case $y=1$ the solution smoothness

$$
u(\cdot, y) \in M=\left\{u(\cdot, y) \in X \mid u \text { obeys }(5.1),\|u(\cdot, 1)\|_{p} \leq E\right\},
$$

where $\|\cdot\|_{p}$ is the norm in the Sobolev space $H^{p}(\mathbb{R})$ of order $p \geq 0$, that is,

$$
\|h\|_{p}:=\left(\int_{\mathbb{R}}|\widehat{h}(\xi)|^{2}\left(1+\xi^{2}\right)^{p} \mathrm{~d} \xi\right)^{1 / 2} \quad \text { with } \widehat{h}(\xi)=\frac{1}{\sqrt{2 \pi}} \int_{\mathbb{R}} h(x) e^{-i x \xi} \mathrm{d} x .
$$

In (5.3), $\widehat{h}(\xi)=\mathcal{F}(h(x))$ is the Fourier transform of the function $h(x)$. Now our aim is to find a stability estimate $\|f\| \leq \beta(\|g\|)$, or equivalently, $\|\widehat{f}\| \leq \beta(\|\widehat{g}\|)$.

Step 1 (Operator equation formulation of the problem): By the method of Fourier transform we obtain in the frequency space the operator equation

$$
\widehat{A} \widehat{f}=\widehat{g} \quad \text { with } \quad \hat{A} \widehat{f}:=\frac{1}{\cosh (y \xi)} \widehat{f}(\xi) .
$$

Step 2 (Deriving the index function $\varphi$ ): Now we ask the question if our smoothness assumption is equivalent to

$$
u(\cdot, y) \in M_{\varphi, E}=\left\{u(\cdot, y) \in X \mid u(\cdot, y)=\left[\varphi\left(A^{*} A\right)\right]^{1 / 2} v,\|v\| \leq E\right\}
$$

with some index function $\varphi=\varphi(\lambda)$. Some formal computations show that there holds equality if $\varphi:(0,1] \rightarrow(0,1]$ is implicitly given by

$$
\varphi\left(1 / \cosh ^{2}(\xi y)\right)=\left(1+\xi^{2}\right)^{-p} \cosh ^{2}(\xi y) / \cosh ^{2} \xi, \quad 0 \leq \xi<\infty .
$$

For showing that $\varphi$ is an index function we rewrite (5.5) into parameter representation $\lambda(\xi)=1 / \cosh ^{2}(\xi y), \varphi(\xi)=\left(1+\xi^{2}\right)^{-p} \cosh ^{2}(\xi y) / \cosh ^{2} \xi, 0 \leq \xi<\infty$, verify that $\dot{\lambda}(\xi)<0$ and $\dot{\varphi}(\xi)<0$ and obtain that $\varphi^{\prime}(\lambda)>0$. Since in addition $\lim _{\xi \rightarrow \infty} \varphi(\xi)=0$ we conclude that $\varphi$ is an index function.

Step 3 (Deriving the function $\varrho$ and verifying its convexity): From (5.5) we obtain that the function $\varrho:(0,1] \rightarrow(0,1]$ is implicitly given by

$$
\varrho\left(\left(1+\xi^{2}\right)^{-p} \cosh ^{2}(\xi y) / \cosh ^{2} \xi\right)=\left(1+\xi^{2}\right)^{-p} / \cosh ^{2} \xi, \quad 0 \leq \xi<\infty .
$$

This function $\varrho$ can be rewritten into parameter representation (3.1) with

$$
c_{1}=1, \quad c_{2}=1 \quad \text { and } \quad \xi_{0}=0 .
$$

From Corollary 3.4 we have

Proposition 5.1. The function $\varrho(\lambda)$, defined in parameter representation by (3.1) with constants (5.7), is convex for all $(y, p) \in(0,1] \times[0, \infty)$.

Step 4 (Deriving the conditional stability estimate): Due to the preparatory steps $1-3$ we are now able to apply the general Theorem 2.1 and obtain 
Theorem 5.2. On the set $M$ given by (5.2) we have for $y \in(0,1)$ an improved Hölder type conditional stability estimate and for $y=1$ some logarithmic type conditional stability estimate $\|f\| \leq \beta(\|g\|)$ with

$$
\beta(\delta)=E\left(1+\xi_{0}^{2}\right)^{-p / 2} \cosh \left(\xi_{0} y\right) / \cosh \xi_{0}=\delta \cosh \left(\xi_{0} y\right)
$$

where $\xi_{0}$ is the unique solution of the equation $\left(1+\xi^{2}\right)^{-p / 2} / \cosh \xi=\delta / E$. For $\delta \rightarrow 0$ there holds the asymptotic representation

$$
\beta(\delta)=E^{y}\left(\frac{\delta}{2}\right)^{1-y}\left(\ln \frac{1}{\delta}\right)^{-p y}(1+o(1))
$$

Proof (sketch). From (5.6) we obtain that the inverse $\varrho^{-1}$ is given by

$$
\varrho^{-1}\left(\left(1+\xi^{2}\right)^{-p} / \cosh ^{2} \xi\right)=\left(1+\xi^{2}\right)^{-p} \cosh ^{2}(\xi y) / \cosh ^{2} \xi, \quad 0 \leq \xi<\infty .
$$

Hence, (5.8) follows. From (5.10) we obtain that the inverse $\varrho^{-1}$ possesses the asymptotic (explicit) representation

$$
\rho^{-1}(\lambda)=(\lambda / 4)^{1-y}(-\ln \sqrt{\lambda})^{-2 p y}(1+o(1)) \quad \text { for } \lambda \rightarrow 0 .
$$

From (5.11) and (2.2) we obtain (5.9).

\section{Cauchy problem for the Helmholtz equation}

Problems of this kind have been considered, e. g., in $[9,20,56,69]$. They arise, e. g., in optoelectronics, and in particular in laser beam models, see $[4,54,55,57]$.

System equation formulation of the problem: Denote by $r=(x, y)$ the first two variables, let $\Delta u:=u_{x x}+u_{y y}+u_{z z}$, and let $k>0$ denote the wave number. Determine, for any fixed $z \in[0, d)$, the function $f(r):=u(r, z)$ from the data function $g(r):=u(r, d)$ where $u(r, z)$ obeys the following Cauchy problem for the Helmholtz equation

$$
\left.\begin{array}{rlrl}
\Delta u+k^{2} u & =0 & & \text { for } \quad(r, z) \in \mathbb{R}^{2} \times(0, d) \\
u(r, d) & =g(r) & & \text { for } \quad r=(x, y) \in \mathbb{R}^{2} \\
u(\cdot, z) & \in L^{2}\left(\mathbb{R}^{2}\right) & \text { for } \quad z \in(0, d]
\end{array}\right\}
$$

Smoothness assumption: Let $X=L^{2}\left(\mathbb{R}^{2}\right)$ with norm $\|\cdot\|$. For studying conditional stability, we assume for some $E>0$ and $p \geq 0$ in case $z \in(0, d)$ and $p>0$ in case $z=0$ the solution smoothness

$$
u(\cdot, z) \in M=\left\{u(\cdot, z) \in X \mid u \text { obeys }(6.1),\|u(\cdot, 0)\|_{p} \leq E\right\}
$$

where $\|\cdot\|_{p}$ is the norm in the Sobolev space $H^{p}\left(\mathbb{R}^{2}\right)$ of order $p \geq 0$, that is,

$$
\|w\|_{p}:=\left(\int_{\mathbb{R}^{2}}|\widehat{w}(\xi)|^{2}\left(1+|\xi|^{2}\right)^{p} \mathrm{~d} \xi\right)^{1 / 2} \text { with } \widehat{w}(\xi)=\frac{1}{2 \pi} \int_{\mathbb{R}^{2}} w(r) \mathrm{e}^{-\mathbf{i} r \cdot \xi} \mathrm{d} r
$$

where $\widehat{w}(\xi)=\mathcal{F}(w(r))$ is the Fourier transform of the $2 D$ function $w(r)$ with respect to the variable $r=(x, y)$ and $\mathcal{F} \in \mathcal{L}(X, X)$ is the Fourier operator. Our aim is to find a stability estimate $\|f\| \leq \beta(\|g\|)$, or equivalently, $\|\widehat{f}\| \leq \beta(\|\widehat{g}\|)$. 
Step 1 (Operator equation formulation of the problem): By the method of Fourier transform we obtain in the frequency space the following operator equation $\widehat{A}(z) \widehat{u}(\xi, z)=\widehat{u}(\xi, d)$, or equivalently,

$$
\widehat{A} \widehat{f}=\widehat{g}, \quad \widehat{A} \widehat{f}=\frac{1}{\cosh \left((d-z) \sqrt{|\xi|^{2}-k^{2}}\right)} \widehat{f}(\xi)
$$

with $\xi=\left(\xi_{1}, \xi_{2}\right),|\xi|^{2}=\xi_{1}^{2}+\xi_{2}^{2}$ and $\widehat{A}(z)=\mathcal{F} A(z) \mathcal{F}^{-1}$. For large wave numbers $k \geq \frac{\pi}{2(d-z)}$ the operator $\widehat{A}(z)$ is unbounded. For deriving conditional stability estimates we therefore use the decomposition idea as outlined in [56, 69]. We decompose $\mathbb{R}^{2}$ into $\mathbb{R}^{2}=I \cup W$ and call $I=\left\{\xi \in \mathbb{R}^{2}|| \xi \mid \geq k\right\}$ the ill-posed part and $W=\left\{\xi \in \mathbb{R}^{2}|| \xi \mid \leq k\right\}$ the well-posed part. Next, we decompose the space $X$ into the direct sum

$$
X=X_{1} \oplus X_{2} \quad \text { with } \quad X_{1}=L^{2}(I) \quad \text { and } \quad X_{2}=L^{2}(W) .
$$

We introduce $P_{1}, P_{2}$ as the orthoprojections onto $X_{1}$ and $X_{2}$, respectively, and decompose, for any fixed $z \in[0, d)$, the element $\widehat{f}(\xi)$ into the sum

$$
\widehat{f}(\xi)=\widehat{f}_{1}(\xi)+\widehat{f}_{2}(\xi) \quad \text { with } \quad \widehat{f}_{1}(\xi)=P_{1} \widehat{f}(\xi) \quad \text { and } \quad \widehat{f}_{2}(\xi)=P_{2} \widehat{f}(\xi)
$$

This decomposition allows to decompose the above operator equation in the frequency domain into two separate problems, one ill-posed problem

$$
\widehat{A}_{1} \widehat{f}_{1}(\xi):=\frac{1}{\cosh \left((d-z) \sqrt{|\xi|^{2}-k^{2}}\right)} \widehat{f}_{1}(\xi)=\widehat{g}_{1}(\xi), \quad \widehat{A}_{1}: X_{1} \rightarrow X_{1}, \widehat{g}_{1}:=P_{1} \widehat{g},
$$

and, by using $\cosh i z=\cos z$, one well-posed problem

$$
\widehat{A}_{2} \widehat{f}_{2}(\xi):=\frac{1}{\cos \left((d-z) \sqrt{k^{2}-|\xi|^{2}}\right)} \widehat{f}_{2}(\xi)=\widehat{g}_{2}(\xi), \widehat{A}_{2}: X_{2} \rightarrow X_{2}, \widehat{g}_{2}:=P_{2} \widehat{g} .
$$

The well-posed problem is stable and, since $\left\|\widehat{A}_{2}^{-1}\right\| \leq 1$, we have $\left\|\widehat{f}_{2}\right\| \leq\left\|\widehat{g}_{2}\right\|$. It remains to find a stability estimate $\left\|\widehat{f}_{1}\right\| \leq \beta\left(\left\|\widehat{g}_{1}\right\|\right)$ for the ill-posed part.

Step 2 (Deriving the index function $\varphi$ for the ill-posed part): Since $\widehat{u}(\cdot, 0)=$ $\widehat{A}(0)^{-1} \widehat{A}(z) \widehat{f}(\cdot)$ we obtain that the smoothness assumption $\|u(\cdot, 0)\|_{p} \leq E$ is equivalent to $\left\|\left(1+|\cdot|^{2}\right)^{p / 2} \widehat{A}(0)^{-1} \widehat{A}(z) \widehat{f}(\cdot)\right\|_{L^{2}\left(\mathbb{R}^{2}\right)} \leq E$. We conclude that

$$
\int_{I}\left(1+|\xi|^{2}\right)^{p} \frac{\cosh ^{2}\left(d \sqrt{|\xi|^{2}-k^{2}}\right)}{\cosh ^{2}\left((d-z) \sqrt{|\xi|^{2}-k^{2}}\right)}\left|\widehat{f}_{1}(\xi)\right|^{2} \mathrm{~d} \xi \leq E_{1}^{2}
$$

where $I=\left\{\xi \in \mathbb{R}^{2}|| \xi \mid \geq k\right\}$ is the ill-posed part, $\widehat{f}_{1}=P_{1} \widehat{f}$ and $E_{1} \leq E$. Now we ask the question if the set of functions $\widehat{f}_{1}(\xi)$ that obey $(6.5)$ is equivalent to

$$
M_{\varphi, E_{1}}=\left\{\widehat{f}_{1}(\xi) \in X_{1} \mid\left\|\left[\varphi\left(\widehat{A}_{1}^{*} \widehat{A}_{1}\right)\right]^{-1 / 2} \widehat{f}_{1}(\xi)\right\|_{L^{2}(I)} \leq E_{1}\right\}
$$


with some index function $\varphi=\varphi(\lambda)$. Some formal computations show that both sets are equal if $\varphi:(0,1] \rightarrow(0,1]$ is given (in parameter representation) by

$$
\left.\begin{array}{l}
\lambda(t)=\frac{1}{\cosh ^{2}\left((d-z) \sqrt{t^{2}-k^{2}}\right)} \\
\varphi(t)=\frac{\cosh ^{2}\left((d-z) \sqrt{t^{2}-k^{2}}\right)}{\left(1+t^{2}\right)^{p} \cosh ^{2}\left(d \sqrt{t^{2}-k^{2}}\right)}
\end{array}\right\} \quad(k \leq t<\infty) .
$$

For showing that $\varphi$ is an index function we verify that $\dot{\lambda}(t)<0$ and $\dot{\varphi}(t)<0$ and obtain that $\varphi^{\prime}(\lambda)>0$. Since in addition $\lim _{t \rightarrow \infty} \varphi(t)=0$ we conclude that $\varphi$ is an index function.

Step 3 (Deriving the function @ for the ill-posed part and verify its convexity): From $(6.7)$ we obtain that the function $\varrho:(0,1] \rightarrow(0,1]$ is given (in parameter representation) by

$$
\left.\begin{array}{l}
\lambda(t)=\frac{\cosh ^{2}\left((d-z) \sqrt{t^{2}-k^{2}}\right)}{\left(1+t^{2}\right)^{p} \cosh ^{2}\left(d \sqrt{t^{2}-k^{2}}\right)} \\
\varrho(t)=\frac{1}{\left(1+t^{2}\right)^{p} \cosh ^{2}\left(d \sqrt{t^{2}-k^{2}}\right)}
\end{array}\right\} \quad(k \leq t<\infty) .
$$

We substitute in (6.8) $d \sqrt{t^{2}-k^{2}}=\xi$ and see that $\varrho$ has the form (3.1) with

$$
c_{1}=d^{2 p}, \quad c_{2}=d^{2}\left(1+k^{2}\right), \quad y=(d-z) / d \quad \text { and } \quad \xi_{0}=0 .
$$

From Corollary 3.4 we have

Proposition 6.1. The function $\varrho(\lambda)$ defined in parameter representation by (6.8) is convex if and only if (3.10) holds with $\left(c_{2}, y\right)$ defined by (6.9). This convexity condition is valid for all $(y, p) \in(0,1] \times[0, \infty)$ if and only if $d^{2}\left(1+k^{2}\right) \geq 3 / 4$.

If the condition $d^{2}\left(1+k^{2}\right) \geq 3 / 4$ is violated, then, by Corollary 3.4 , there is some sub-range (where $p$ is close to zero and $y$ is close to one) of the range $(y, p) \in(0,1] \times[0, \infty)$ where convexity of $\varrho$ is violated.

Step 4 (Deriving the conditional stability estimate): Due to the preparatory steps $1-3$ we are now able to apply the general Theorems 2.1 and 2.3 and obtain

Theorem 6.2. Let $\varrho(\lambda)$ be convex. Then, on the set (6.6) we have for the ill-posed part for any $z \in(0, d)$ an improved Hölder type conditional stability estimate and for $z=0$ some logarithmic type stability estimate $\left\|f_{1}\right\| \leq \beta\left(\left\|g_{1}\right\|\right)$ with

$$
\beta(\delta)=E_{1} \frac{\cosh \left((d-z) \sqrt{t_{0}^{2}-k^{2}}\right)}{\left(1+t_{0}^{2}\right)^{p / 2} \cosh \left(d \sqrt{t_{0}^{2}-k^{2}}\right)}
$$

where $t_{0}$ is the unique solution of the equation $\left(1+t^{2}\right)^{p / 2} \cosh \left(d \sqrt{t^{2}-k^{2}}\right)=E_{1} / \delta$. For $\delta \rightarrow 0$ there holds the asymptotic representation

$$
\beta(\delta)=E_{1}^{1-z / d}\left(\frac{\delta}{2}\right)^{z / d}\left[\frac{1}{d} \ln \frac{1}{\delta}\right]^{-p(1-z / d)}(1+o(1)) .
$$


If $\varrho(\lambda)$ is not convex, then (6.10) and (6.11) hold true with an additional factor $\sqrt{2}$ on the right hand side.

Proof (sketch). From (6.8) we obtain that the inverse $\varrho^{-1}$ is given by

$$
\left.\begin{array}{l}
\lambda(t)=\frac{1}{\left(1+t^{2}\right)^{p} \cosh ^{2}\left(d \sqrt{t^{2}-k^{2}}\right)} \\
\varrho^{-1}(t)=\frac{\cosh ^{2}\left((d-z) \sqrt{t^{2}-k^{2}}\right)}{\left(1+t^{2}\right)^{p} \cosh ^{2}\left(d \sqrt{t^{2}-k^{2}}\right)}
\end{array}\right\} \quad(k \leq t<\infty) .
$$

From this representation and formula (2.2) we obtain (13.7). From (6.12) we have

$$
\varrho^{-1}(\lambda)=\left(\frac{\lambda}{2}\right)^{z / d}\left(\frac{1}{2 d} \ln \frac{1}{\lambda}\right)^{-2 p(1-z / d)}(1+o(1)) \text { for } \lambda \rightarrow 0 .
$$

From this asymptotic representation and (2.2) we obtain (6.11).

Remark 6.3. From the above two stability estimates (6.11) for the ill-posed part and $\left\|f_{2}\right\| \leq\left\|g_{2}\right\|$ for the well-posed part we obtain due to the Pythagoras Theorem that on the set $M$ defined by (6.2) we have $\|f\| \leq \beta(\|g\|)$ with

$$
\beta(\delta)=E^{1-z / d}\left(\frac{\delta}{2}\right)^{z / d}\left[\frac{1}{d} \ln \frac{1}{\delta}\right]^{-p(1-z / d)}(1+o(1)) .
$$

We further remark that this asymptotic conditional stability function is independent on the wave number $k$.

\section{Backward heat conduction}

Problems of this type have been under consideration, e.g., in the papers [16, $50,61,62,72,74]$ and are one of the classical ill-posed problems with various engineering applications, see, e.g., $[2,5,48]$ and the references cited there.

System equation formulation of the problem: Determine, for any fixed $t \in[0, T)$, the function $f(x):=u(x, t), x \in \Omega \subset \mathbb{R}^{n}$, from the data function $g(x):=u(x, T)$ where $u(x, t)$ obeys the following backward heat equation problem

$$
\left.\begin{array}{rlrl}
u_{t}+L u & =0 & & \text { for } \quad x \in \Omega \subset \mathbb{R}^{n}, 0<t<T \\
u(x, T) & =g(x) & \text { for } \quad x \in \Omega \subset \mathbb{R}^{n}
\end{array}\right\}
$$

and $L: D(L) \subset X \rightarrow X$ denotes a linear densely defined self adjoint and positive definite operator in $X=L^{2}(\Omega)$ with eigenvalues

$$
1=l_{1} \leq l_{2} \leq \ldots \leq l_{i} \leq \ldots, \quad l_{i} \rightarrow \infty \quad \text { for } \quad i \rightarrow \infty
$$

and eigenelements $\left\{u_{i}\right\}$ that form an orthonormal basis in $X$. For a simple example for $L$, see Section 3.

Smoothness assumption: For studying conditional stability, we assume that $\left\|L^{p / 2} u(x, 0)\right\| \leq E$ with some $E>0$ and $p \geq 0$ in case $t \in(0, T)$ and $p>0$ in case $t=0$, that is, we assume

$$
u(\cdot, t) \in M=\left\{u(\cdot, t) \in X \mid u \text { obeys }(7.1),\left\|L^{p / 2} u(\cdot, 0)\right\| \leq E\right\}
$$

and ask for a stability estimate $\|f\| \leq \beta(\|g\|)$. 
Step 1 (Operator equation formulation of the problem): By the method of separation of variables we obtain from $(7.1)$ the operator equation $A(t) u(\cdot, t)=u(\cdot, T)$, or equivalently,

$$
A f=g \quad \text { with } \quad \text { Af }=\sum_{n=1}^{\infty} \sigma_{n}\left(f, u_{n}\right) u_{n} \quad \text { and } \quad \sigma_{n}=\mathrm{e}^{-(T-t) l_{n}} .
$$

Hence, $\sigma_{n}$ are the eigenvalues and $u_{n}$ the corresponding eigenelements of the selfadjoint and compact operator $A \in \mathcal{L}(X)$.

Step 2 (Deriving the index function $\varphi$ ): Now we ask the question if the set $M$ is equivalent to some general source set

$$
M_{\varphi, E}=\left\{f \in X \mid f=\left[\varphi\left(A^{*} A\right)\right]^{1 / 2} v,\|v\| \leq E\right\}
$$

with some index function $\varphi=\varphi(\lambda)$. Some formal computations show that both sets $M$ and $M_{\varphi, E}$ are equal if $\varphi:\left(0, \mathrm{e}^{-2(T-t)}\right] \rightarrow\left(0, \mathrm{e}^{-2 t}\right]$ is implicitly given by

$$
\varphi\left(\mathrm{e}^{-2 l(T-t)}\right)=l^{-p} \mathrm{e}^{-2 l t}, \quad 1 \leq l<\infty .
$$

The function $\varphi$ defined by (7.4) is an index function and has the explicit form $\varphi(\lambda)=\lambda^{t /(T-t)}\left[\frac{1}{2(T-t)} \ln \frac{1}{\lambda}\right]^{-p}$.

Step 3 (Deriving the function $\varrho$ and verifying its convexity): From (7.4) we obtain that the function $\varrho:\left(0, \mathrm{e}^{-2 t}\right] \rightarrow\left(0, \mathrm{e}^{-2 T}\right]$ is implicitly given by

$$
\varrho\left(l^{-p} \mathrm{e}^{-2 l t}\right)=l^{-p} \mathrm{e}^{-2 l T}, \quad 1 \leq l<\infty .
$$

We substitute in (7.5) $2 T l=\xi$ and see that $\varrho$ defined by (7.5) has the special form (3.11) with constants

$$
c_{1}=(2 T)^{p}, \quad c_{2}=0, \quad c_{3}=1, \quad \xi_{0}=2 T \quad \text { and } \quad z=t / T .
$$

From Proposition 3.5 we conclude

Proposition 7.1. The function $\varrho(\lambda)$, defined in parameter representation by (3.11) with constants (7.6), is convex if and only if

$$
p^{2}+p[2(T+t)-1]+4 t T \geq 0 .
$$

This inequality is valid

(i) for all $t \in[0, T)$ if and only if $p^{2}+p[2 T-1] \geq 0$,

(ii) for all $p \geq 0$ if and only if $\sqrt{T}+\sqrt{t} \geq 1 / \sqrt{2}$,

(iii) for all $t \in[0, T)$ and all $p \geq 0$ if and only if $T \geq 1 / 2$.

Proof. For the function $\psi$ defined by $(3.13)$ we have $\psi(\xi)=1 / \xi$ and $\dot{\psi}(\xi)=-1 / \xi^{2}$. We apply Proposition 3.5 and obtain that $\varrho(\lambda)$ is convex if and only if

$$
p^{2}+p[\xi(z+1)-1]+\xi^{2} z \geq 0 \text { for all } \xi \in\left[\xi_{0}, \infty\right),
$$

which holds true if and only if (7.7) is valid. From (7.7) we immediately obtain the statement (i) of the proposition. For the proof of (ii) we distinguish two cases $2(T+t)-1 \geq 0$ and $2(T+t)-1<0$. In the first case, $(7.7)$ holds true for all $p \geq 0$. In the second case with $2(T+t)-1<0,(7.7)$ holds true if and only if

$$
[2(T+t)-1]^{2} \leq 16 t T, \quad \text { or equivalently, } \quad \sqrt{T}+\sqrt{t} \geq 1 / \sqrt{2} .
$$


From the both cases we obtain (ii). The statement (iii) follows from (ii).

Remark 7.2. If the convexity condition (7.7) is violated, then there exists some $\lambda^{*}<\lambda\left(\xi_{0}\right)$ with property that

(i) $\varrho(\lambda)$ is convex on the restricted $\lambda$-range $\lambda \in\left(0, \lambda^{*}\right]$ and

(ii) $\varrho(\lambda)$ is not convex on the $\lambda$-range $\lambda \in\left(\lambda^{*}, \lambda\left(\xi_{0}\right)\right]$.

The argument $\lambda^{*}$ can be computed explicitly by first computing the (unique) positive solution $\xi=\xi^{*}$ of the equation $p^{2}+p[\xi(z+1)-1]+\xi^{2} z=0$ and then computing $\lambda^{*}:=\lambda\left(\xi^{*}\right)$. The both statements (i) and (ii) follow from $\dot{\lambda}(\xi)<0$ and the facts that the convexity condition (7.8) is satisfied for $\xi \in\left[\xi^{*}, \infty\right)$ and violated for $\xi \in\left[\xi_{0}, \xi^{*}\right)$. Let us consider the special case where $t=0$. Then we conclude from (7.8) that $\varrho(\lambda)$ is convex if and only if

$$
p+\xi-1 \geq 0 \text { for all } \xi \in\left[\xi_{0}, \infty\right)
$$

Assume now that this convexity condition is violated. Then, computing $\xi^{*}$ and $\lambda^{*}$ yields $\xi^{*}=1-p$ and $\lambda^{*}=\left(\frac{2 T}{1-p}\right)^{p}<1=\lambda\left(\xi_{0}\right)$.

Step 4 (Deriving the conditional stability estimate): Due to the preparatory steps $1-3$ we are now able to apply the general Theorems 2.1 and 2.3 and obtain

Theorem 7.3. Let the convexity condition (7.7) be valid. Then, on the set $M$ given by (7.2) we have for $t \in(0, T)$ an improved Hölder type conditional stability estimate and for $t=0$ some logarithmic type conditional stability estimate $\|f\| \leq$ $\beta(\|g\|)$ with

$$
\beta(\delta)=E l_{0}^{-p / 2} \mathrm{e}^{-l_{0} t}=E^{1-t / T} \delta^{t / T} l_{0}^{-p(T-t) /(2 T)}
$$

where $l_{0}$ is the unique positive solution of the equation $l^{-p / 2} \mathrm{e}^{-l T}=\delta / E$. For $\delta \rightarrow 0$ there holds the asymptotic representation

$$
\beta(\delta)=E^{1-t / T} \delta^{t / T}\left(\frac{1}{T} \ln \frac{1}{\delta}\right)^{-p(T-t) /(2 T)}(1+o(1)) .
$$

If the convexity condition (7.7) is violated, then (7.9) and (7.10) hold true with an additional factor $\sqrt{2}$ on the right hand side.

Proof (sketch). From (7.5) we obtain that the inverse $\varrho^{-1}$ is implicitly given by

$$
\varrho^{-1}\left(l^{-p} \mathrm{e}^{-2 l T}\right)=l^{-p} \mathrm{e}^{-2 l t}, \quad 1 \leq l<\infty .
$$

Hence, (7.9) follows. From $l_{0}^{-p / 2} \mathrm{e}^{-l_{0} T}=\delta / E$ we have

$$
l_{0}=\left(\frac{1}{T} \ln \frac{1}{\delta}\right)(1+o(1)) \quad \text { for } \quad \delta \rightarrow 0
$$

From this asymptotic representation and (7.9) we obtain (7.10). 


\section{Fractional backward heat conduction in a strip}

This problem is taken from [82]. For the special case $\alpha=2$ and $\mu=0$, see [73]. Fractional models are required for modeling complex systems in nature with anomalous dynamics arising in biology, chemistry, physics, geology, astrophysics or social sciences, and in particular in transport of chemical contaminations through water around rocks, dynamics of viscoelastic materials as polymers, diffusion of pollution in the atmosphere, diffusion processes involving cells, signal theory, control theory or electromagnetic theory, see [36]. In most of the above-mentioned applications, the kind of anomalous processes has a macroscopic complex behavior and its dynamics cannot be characterized by classical derivative models.

System equation formulation of the problem: Determine, for any fixed $t \in[0, T)$, the function $f(x):=u(x, t)$ from the data function $g(x):=u(x, T)$ where $u(x, t)$ obeys the following fractional heat equation in the strip $0<t<T$ :

$$
\left.\begin{array}{rlrl}
u_{t}-{ }_{x} D_{\mu}^{\alpha} u & =0 & & \text { for } \quad x \in \mathbb{R}, 0<t<T \\
\left.u\right|_{x \rightarrow \pm \infty} & =0 & & \text { for } t \in(0, T] \\
u(x, T) & =g(x) & & \text { for } \quad x \in \mathbb{R}
\end{array}\right\} .
$$

In (8.1), ${ }_{x} D_{\mu}^{\alpha} u$ is the Riesz-Feller fractional derivative of the function $u$ (with respect to $x)$ of order $\alpha(0<\alpha \leq 2)$ and skewness $\mu(|\mu| \leq \min \{\alpha, 2-\alpha\}, \mu \neq \pm 1)$, see $[23,36]$, which is defined via Fourier transform by

$$
\mathcal{F}\left({ }_{x} D_{\mu}^{\alpha} h(x)\right)=-\theta(\xi) \widehat{h}(\xi), \quad \theta(\xi)=|\xi|^{\alpha} \mathrm{e}^{\mathrm{i} \operatorname{sgn}(\xi) \mu \pi / 2} .
$$

We note that for $\alpha=2$ and $\mu=0$ we have ${ }_{x} D_{\mu}^{\alpha} h(x)=\frac{\mathrm{d}^{2}}{\mathrm{~d} x^{2}} h(x)$.

Smoothness assumption: Let $X=L^{2}(\mathbb{R})$ with norm $\|\cdot\|$. For studying conditional stability, we assume for some $E>0$ and $p \geq 0$ in case $t \in(0, T)$ and $p>0$ in case $t=0$ the solution smoothness $\|u(x, 0)\|_{p} \leq E$ where $\|\cdot\|_{p}$ is the norm in the Sobolev space $H^{p}(\mathbb{R})$ of order $p \geq 0$, see (5.3). Hence, we assume

$$
u(\cdot, t) \in M=\left\{u(\cdot, t) \in X \mid u \text { obeys }(8.1),\|u(\cdot, 0)\|_{p} \leq E\right\}
$$

and ask for a stability estimate $\|f\| \leq \beta(\|g\|)$, or equivalently, $\|\widehat{f}\| \leq \beta(\|\widehat{g}\|)$.

Step 1 (Operator equation formulation of the problem): By the method of Fourier transform we obtain in the frequency space the operator equation

$$
\widehat{A} \widehat{f}=\widehat{g} \quad \text { with } \quad \widehat{A} \widehat{f}:=\mathrm{e}^{-\theta(\xi)(T-t)} \widehat{f}(\xi) .
$$

Step 2 (Deriving the index function $\varphi$ ): Now we ask the question if our smoothness assumption is equivalent to

$$
u(\cdot, t) \in M_{\varphi, E}=\left\{u(\cdot, t) \in X \mid u(\cdot, t)=\left[\varphi\left(A^{*} A\right)\right]^{1 / 2} v,\|v\| \leq E\right\}
$$

with some index function $\varphi=\varphi(\lambda)$. Some formal computations show that there holds equality if $\varphi:(0,1] \rightarrow(0,1]$ is implicitly given by

$$
\varphi\left(\mathrm{e}^{-2|\xi|^{\alpha}(T-t) \cos \mu \pi / 2}\right)=\left(1+|\xi|^{2}\right)^{-p} \mathrm{e}^{-2|\xi|^{\alpha} t \cos \mu \pi / 2}, \quad \xi \in \mathbb{R} .
$$

It can be shown that the function $\varphi$ defined by (8.4) is an index function. 
Step 3 (Deriving the function $\varrho$ and verifying its convexity): From (8.4) we obtain that the function $\varrho:(0,1] \rightarrow(0,1]$ is given by

$$
\varrho\left(\left(1+|\xi|^{2}\right)^{-p} \mathrm{e}^{-2|\xi|^{\alpha} t \cos (\mu \pi / 2)}\right)=\left(1+|\xi|^{2}\right)^{-p} \mathrm{e}^{-2|\xi|^{\alpha} T \cos (\mu \pi / 2)}, \quad \xi \in \mathbb{R} .
$$

We substitute in (8.5) $2 T|\xi|^{\alpha} \cos (\mu \pi / 2)=r$ and then $r=\xi$ and see that $\varrho$ defined by (8.5) has the special form (3.11) with constants

$$
\left.\begin{array}{ll}
c_{1}=(2 T \cos (\mu \pi / 2))^{2 p / \alpha}, & c_{2}=(2 T \cos (\mu \pi / 2))^{2 / \alpha} \\
c_{3}=2 / \alpha, \quad \xi_{0}=0, & z=t / T
\end{array}\right\} .
$$

From Proposition 3.5 and Corollary 3.6 we conclude

Proposition 8.1. The function $\varrho(\lambda)$, defined in parameter representation by (3.11) with constants (8.6), is convex if and only if (3.12) holds true with constants $\left(c_{2}, c_{3}, \xi_{0}, z\right)$ given by (8.6). This convexity condition is valid for all $(t, p) \in$ $[0, T) \times[0, \infty)$ and all $\alpha \in(0,2]$ if and only if $2 T \cos (\mu \pi / 2) \geq 1$.

Proof. From Corollary 3.6 we have that $\varrho(\lambda)$ is convex for all $(t, p) \in[0, T) \times[0, \infty)$ if and only if $f_{1}(\alpha) \leq f_{2}(\alpha), \alpha \in(0,2]$, where

$$
f_{1}(\alpha)=\sup _{\xi \in[0, \infty)} \frac{\xi^{2 / \alpha}(1-\xi)}{\xi+2 / \alpha-1}, \quad f_{2}(\alpha)=(2 T \cos (\mu \pi / 2))^{2 / \alpha}
$$

The function $f_{1}$ is monotonically increasing with $\lim _{\alpha \rightarrow 0} f_{1}(\alpha)=0$ and $f_{1}(2)=1$. Consider now two cases $2 T \cos (\mu \pi / 2)<1$ and $2 T \cos (\mu \pi / 2) \geq 1$. In the first case, $f_{2}(\alpha)$ is increasing with $\lim _{\alpha \rightarrow 0} f_{2}(\alpha)=0$ and $f_{2}(2)=2 T \cos (\mu \pi / 2)<1$. Hence, the convexity condition $f_{1}(\alpha) \leq f_{2}(\alpha)$ is not valid for all $\alpha \in(0,2]$. In the second case with $2 T \cos (\mu \pi / 2) \geq 1$ there holds $f_{2}(\alpha) \geq 1$ for all $\alpha \in(0,2]$. Hence, in the second case, the convexity condition $f_{1}(\alpha) \leq f_{2}(\alpha)$ is valid for all $\alpha \in(0,2]$.

Step 4 (Deriving the conditional stability estimate): Due to the preparatory steps $1-3$ we are now able to apply the general Theorems 2.1 and 2.3 and obtain

Theorem 8.2. Let $\varrho(\lambda)$ be convex. Then, on the set $M$ given by (8.2) we have for $t \in(0, T)$ an improved Hölder type conditional stability estimate and for $t=0$ some logarithmic type conditional stability estimate $\|f\| \leq \beta(\|g\|)$ with

$$
\beta(\delta)=E\left(1+r_{0}^{2 / \alpha}\right)^{-p / 2} \mathrm{e}^{-r_{0} t \cos (\mu \pi / 2)}=E^{1-t / T} \delta^{t / T}\left(1+r_{0}^{2 / \alpha}\right)^{-p(T-t) /(2 T)}
$$

where $r_{0}$ is the unique solution of the equation $\left(1+r^{2 / \alpha}\right)^{-p / 2} \mathrm{e}^{-r T \cos (\mu \pi / 2)}=\delta / E$. For $\delta \rightarrow 0$ there holds the asymptotic representation

$$
\beta(\delta)=E^{1-t / T} \delta^{t / T}\left(\frac{1}{T \cos (\mu \pi / 2)} \ln \frac{1}{\delta}\right)^{-p(1-t / T) / \alpha}(1+o(1)) .
$$

If $\varrho(\lambda)$ is not convex, then (8.7) and (8.8) hold true with an additional factor $\sqrt{2}$ on the right hand side. 
Proof (sketch). From (8.5) we obtain that the inverse $\varrho^{-1}$ is implicitly given by

$$
\varrho\left(\left(1+r^{2 / \alpha}\right)^{-p} \mathrm{e}^{-2 r T \cos (\mu \pi / 2)}\right)=\left(1+r^{2 / \alpha}\right)^{-p} \mathrm{e}^{-2 r t \cos (\mu \pi / 2)}, \quad 0 \leq r<\infty .
$$

Hence, (8.7) follows. From $\left(1+r_{0}^{2 / \alpha}\right)^{-p / 2} \mathrm{e}^{-r_{0} T \cos (\mu \pi / 2)}=\delta / E$ we have

$$
r_{0}=\left(\frac{1}{T \cos (\mu \pi / 2)} \ln \frac{1}{\delta}\right)(1+o(1)) \quad \text { for } \quad \delta \rightarrow 0 .
$$

From this asymptotic representation and (8.7) we obtain (8.8).

\section{Backward heat conduction in the plane}

This problem is taken from [3]. We recommend this book also for engineering applications and further references.

System equation formulation of the problem: Let $r=(x, y)$ and $\Delta u=u_{x x}+u_{y y}$. Determine, for any fixed $t \in[0,1)$, the function $f(r):=u(r, t)$ from the data function $g(r):=u(r, 1)$ where $u$ obeys the following heat equation problem

$$
\left.\begin{array}{lll}
u_{t}-\Delta u=0 & \text { for } & (r, t) \in \mathbb{R}^{2} \times \mathbb{R} \\
u(r, 1)=g(r) & \text { for } \quad r \in \mathbb{R}^{2} \\
u(\cdot, t) \in L^{2}\left(\mathbb{R}^{2}\right) & \text { for } \quad t \in \mathbb{R}
\end{array}\right\}
$$

Smoothness assumption: Let $X=L^{2}\left(\mathbb{R}^{2}\right)$ with norm $\|\cdot\|$. For studying conditional stability, we assume for some $E>0$ and $p \geq 0$ in case $t \in(0,1)$ and $p>0$ in case $t=0$ the solution smoothness $\|u(r, 0)\|_{p} \leq E$ where $\|\cdot\|_{p}$ is the norm in the Sobolev space $H^{p}\left(\mathbb{R}^{2}\right)$ of order $p \geq 0$, see (6.3). That is, we assume the solution smoothness

$$
u(\cdot, t) \in M=\left\{u(\cdot, t) \in X \mid u \text { obeys }(9.1),\|u(\cdot, 0)\|_{p} \leq E\right\}
$$

and ask for a stability estimate $\|f\| \leq \beta(\|g\|)$, or equivalently, $\|\widehat{f}\| \leq \beta(\|\widehat{g}\|)$.

Step 1 (Operator equation formulation of the problem): Let $\mathcal{F} \in \mathcal{L}(X, X)$ be the Fourier operator and $\widehat{u}(\xi, t)$ be the Fourier transform of $u(r, t)$ with respect to the variable $r=(x, y)$. Due to $\mathcal{F}\left(u_{x x}(r, t)\right)=-\xi_{1}^{2} \widehat{u}(\xi, t)$ and $\mathcal{F}\left(u_{y y}(r, t)\right)=-\xi_{2}^{2} \widehat{u}(\xi, t)$, $\xi=\left(\xi_{1}, \xi_{2}\right)$, problem (9.1) attains in the frequency space the form

$$
\widehat{u}_{t}(\xi, t)+|\xi|^{2} \widehat{u}(\xi, t)=0, \quad \widehat{u}(\xi, 1)=\widehat{g}(\xi), \quad(\xi, t) \in \mathbb{R}^{2} \times \mathbb{R} .
$$

Solving this final value problem for an ordinary differential eqution yields the operator equation $\widehat{A}(t) \widehat{u}(\xi, t)=\widehat{u}(\xi, 1)$, or equivalently,

$$
\widehat{A} \hat{f}=\widehat{g} \quad \text { with } \quad \widehat{A} \widehat{f}=\mathrm{e}^{-(1-t)|\xi|^{2}} \widehat{f}(\xi) .
$$

From (9.3) we have that both $A$ and $\widehat{A}$ are linear, nonnegative, self-adjoint, injective and bounded operators with non-closed range where $\|A\|=\|\widehat{A}\|=1$.

Step 2 (Deriving the index function $\varphi$ ): Now we ask the question if the set $M$ is equivalent to some general source set

$$
M_{\varphi, E}=\left\{u(\cdot, t) \in X \mid u(\cdot, t)=\left[\varphi\left(A^{*} A\right)\right]^{1 / 2} v,\|v\| \leq E\right\}
$$


with some index function $\varphi=\varphi(\lambda)$. Some formal computations show that both sets (9.2) and (9.4) are equal if $\widehat{A}^{*} \widehat{A} \varphi\left(\widehat{A}^{*} \widehat{A}\right)=\left(1+|\xi|^{2}\right)^{-p} \widehat{A}_{0}^{*} \widehat{A}_{0}$ with $\widehat{A}_{0}=\widehat{A}(0)$, or equivalently, if $\varphi(\lambda):(0,1] \rightarrow(0,1]$ is implicitly given by

$$
\varphi\left(\mathrm{e}^{-2(1-t)|\xi|^{2}}\right)=\left(1+|\xi|^{2}\right)^{-p} \mathrm{e}^{-2 t|\xi|^{2}}, \quad \xi \in \mathbb{R}^{2} .
$$

It can be shown that the function $\varphi$ defined by (9.5) is an index function.

Step 3 (Deriving the function @ and verifying its convexity): By using $\varrho(\varphi(\lambda))=$ $\lambda \varphi(\lambda)$ we obtain that $\varrho\left(\varphi\left(\widehat{A}^{*} \widehat{A}\right)\right)=\left(1+|\xi|^{2}\right)^{-p} \widehat{A}_{0}^{*} \widehat{A}_{0}$. From this representation we obtain that $\varrho$ is implicitly given by

$$
\varrho\left(\left(1+|\xi|^{2}\right)^{-p} \mathrm{e}^{-2 t|\xi|^{2}}\right)=\left(1+|\xi|^{2}\right)^{-p} \mathrm{e}^{-2|\xi|^{2}}, \quad \xi \in \mathbb{R}^{2} .
$$

We substitute in (9.6) $2|\xi|^{2}=r$ and then $r=\xi$ and see that $\varrho$ defined by (9.6) has the special form (3.11) with constants

$$
c_{1}=2^{p}, \quad c_{2}=2, \quad c_{3}=1, \quad \xi_{0}=0 \quad \text { and } \quad z=t .
$$

From Proposition 3.5 we conclude

Proposition 9.1. The function $\varrho(\lambda)$, defined in parameter representation by (3.11) with constants (9.7), is convex for all $(t, p) \in[0,1) \times[0, \infty)$.

Proof. For $\psi$ defined by (3.13) we have $\psi(\xi)=1 /(2+\xi)$ and $\dot{\psi}(\xi)=-1 /(2+\xi)^{2}$. We apply Proposition 3.5 and obtain that $\varrho(\lambda)$ is convex if and only if

$$
p^{2}+p[(2+\xi)(t+1)-1]+(2+\xi)^{2} t \geq 0 \text { for all } \xi \in[0, \infty) .
$$

This convexity condition holds true if and only if $p^{2}+p[2(t+1)-1]+4 t \geq 0$, which is valid for all $(t, p) \in[0,1) \times[0, \infty)$.

Step 4 (Deriving the conditional stability estimate): Due to the preparatory steps $1-3$ we are now able to apply the general Theorem 2.1 and obtain

Theorem 9.2. On the set $M$ given by (9.2) we have for $t \in(0,1)$ an improved Hölder type conditional stability estimate and for $t=0$ some logarithmic type conditional stability estimate $\|f\| \leq \beta(\|g\|)$ with

$$
\beta(\delta)=E\left(1+r_{0}\right)^{-p / 2} \mathrm{e}^{-t r_{0}}=E^{1-t} \delta^{t}\left(1+r_{0}\right)^{-p(1-t) / 2}
$$

where $r_{0}$ is the unique positive solution of the equation $(1+r)^{-p / 2} \mathrm{e}^{-r}=\delta / E$. For $\delta \rightarrow 0$ there holds the asymptotic representation

$$
\beta(\delta)=E^{1-t} \delta^{t}\left(\ln \delta^{-1}\right)^{-p(1-t) / 2}(1+o(1)) .
$$

Proof (sketch). From (9.6) we obtain that the inverse $\varrho^{-1}$ is implicitly given by

$$
\varrho^{-1}\left((1+r)^{-p} \mathrm{e}^{-2 r}\right)=(1+r)^{-p} \mathrm{e}^{-2 t r}, \quad r \in \mathbb{R}^{+} .
$$

Hence, (9.8) follows from formula (2.2). From $\left(1+r_{0}\right)^{-p / 2} \mathrm{e}^{-r_{0}}=\delta / E$ we have

$$
r_{0}=\left(\ln \delta^{-1}\right)(1+o(1)) \quad \text { for } \quad \delta \rightarrow 0 .
$$

From this asymptotic representation and (9.8) we obtain (9.9). 


\section{Non-standard sideways heat conduction}

Problems of this type have been considered, e.g., in the papers $[19,52,71]$. In the special case $b=0$ this problem has been treated in [60]. Different industrial examples for sideways heat conduction problems may be found, e.g., in [5].

System equation formulation of the problem: Determine, for any fixed $x \in[0,1)$, the function $f(t):=u(x, t)$ from the data function $g(t):=u(1, t)$ where $u(x, t)$ obeys the following non-standard sideways heat conduction problem

$$
\left.\begin{array}{rlrl}
u_{t}+b u_{x}-u_{x x} & =0 & & \text { for } \quad x>0, t>0 \\
u(x, 0) & =0 & & \text { for } x \geq 0 \\
u(1, t) & =g(t) & & \text { for } t \geq 0,\left.u(x, t)\right|_{x \rightarrow \infty} \text { bounded }
\end{array}\right\} .
$$

We are in particular interested in the question if the convection term $b u_{x}$ has some influence on the conditional stability of the above problem.

Smoothness assumption: Let $X=L^{2}(\mathbb{R})$ with norm $\|\cdot\|$. Since we are working with Fourier transform with respect to the variable $t$, we extend the domain of the appearing functions with respect to $t$ by defining them to be zero for $t<0$. For studying conditional stability, we assume for some $E>0$ and $p \geq 0$ in case $x \in(0,1)$ and $p>0$ in case $x=0$ the solution smoothness $\|u(0, t)\|_{p} \leq E$ where $\|\cdot\|_{p}$ is the norm in the Sobolev space $H^{p}(\mathbb{R})$ of order $p \geq 0$, see (5.3). That is, we assume the solution smoothness

$$
u(x, \cdot) \in M=\left\{u(x, \cdot) \in X \mid u \text { obeys }(10.1),\|u(0, \cdot)\|_{p} \leq E\right\}
$$

and ask for a stability estimate $\|f\| \leq \beta(\|g\|)$, or equivalently, $\|\widehat{f}\| \leq \beta(\|\widehat{g}\|)$.

Step 1 (Operator equation formulation of the problem): By the method of Fourier transform we obtain in the frequency space the following operator equation $\widehat{A}(x) \widehat{u}(x, \xi)=\widehat{u}(1, \xi)$, or equivalently,

$$
\widehat{A} \hat{f}=\widehat{g} \quad \text { with } \quad \widehat{A} \widehat{f}=\mathrm{e}^{-\theta(\xi)(1-x)} \widehat{f}(\xi)
$$

where $\widehat{A}=\mathcal{F} A \mathcal{F}^{-1}, \mathcal{F}$ is the Fourier operator, $\widehat{u}(x, \xi)$ is the Fourier transform of $u(x, t)$ with respect to the variable $t$ and $\theta=\theta(\xi)$ is given by

$$
\operatorname{Re} \theta=-b / 2+\sqrt{r / 2+b^{2} / 8}, \operatorname{Im} \theta=\operatorname{sign}(\xi) \sqrt{r / 2-b^{2} / 8}, r=\sqrt{\xi^{2}+b^{4} / 16} .
$$

From this representation we obtain that $\left\|A^{*} A\right\|=\mathrm{e}^{-(1-x)(|b|-b)}$. We conclude that the operator $A^{*} A$ has continuous spectrum in the interval $\left(0, \mathrm{e}^{-(1-x)(|b|-b)}\right]$.

Step 2 (Deriving the index function $\varphi$ ): Now we ask the question if the set $M$ is equivalent to some general source set

$$
M_{\varphi, E}=\left\{u(x, \cdot) \in X \mid u(x, \cdot)=\left[\varphi\left(A^{*} A\right)\right]^{1 / 2} v,\|v\| \leq E\right\}
$$

with some index function $\varphi=\varphi(\lambda)$. Some formal computations show that both sets $(10.2)$ and (10.4) are equal if $\widehat{A}^{*} \widehat{A} \varphi\left(\widehat{A}^{*} \widehat{A}\right)=\left(1+\xi^{2}\right)^{-p} \widehat{A}_{0}^{*} \widehat{A}_{0}$ with $\widehat{A}_{0}=\widehat{A}(0)$, or equivalently, if $\varphi(\lambda):\left(0, \mathrm{e}^{-(1-x)(|b|-b)}\right] \rightarrow\left(0, \mathrm{e}^{-x(|b|-b)}\right]$ is implicitly given by

$$
\varphi\left(\mathrm{e}^{-2(1-x) \operatorname{Re} \theta}\right)=\left(1+\xi^{2}\right)^{-p} \mathrm{e}^{-2 x \operatorname{Re} \theta}, \quad 0 \leq \xi<\infty .
$$


For showing that $\varphi$ is an index function for all $b \in \mathbb{R}$ we introduce $g(\xi):=\operatorname{Re} \theta$ and rewrite (10.5) into parameter representation

$$
\lambda(\xi)=\mathrm{e}^{-2(1-x) g(\xi)}, \quad \varphi(\xi)=\left(1+\xi^{2}\right)^{-p} \mathrm{e}^{-2 x g(\xi)}, \quad 0 \leq \xi<\infty,
$$

verify that due to $\dot{g}(\xi)>0$ there holds $\dot{\lambda}(\xi)<0$ and $\dot{\varphi}(\xi)<0$ and obtain that $\varphi^{\prime}(\lambda)>0$. Since in addition $\lim _{\xi \rightarrow \infty} \varphi(\xi)=0$ we conclude that $\varphi$ is an index function.

Step 3 (Deriving the function $\varrho$ and verifying its convexity): From (10.5) we obtain that $\varrho(\lambda):=\lambda \varphi^{-1}(\lambda):\left(0, \mathrm{e}^{-x(|b|-b)}\right] \rightarrow\left(0, \mathrm{e}^{-(|b|-b)}\right]$ is implicitly given by

$$
\varrho\left(\left(1+\xi^{2}\right)^{-p} \mathrm{e}^{-2 x \operatorname{Re} \theta}\right)=\left(1+\xi^{2}\right)^{-p} \mathrm{e}^{-2 \operatorname{Re} \theta}, \quad 0 \leq \xi<\infty .
$$

Following proposition verifies the convexity of the function $\varrho$.

Proposition 10.1. The function @ from (10.6) is convex if and only if

$$
p^{2}+p[(1+t)((1+x) \dot{g}-\ddot{g} / \dot{g})-1]+x(1+t)^{2} \dot{g}^{2} \geq 0 \text { for all } t \in[0, \infty),
$$

where $g$ is defined by $g(t):=-b+\left(\sqrt{4 t+b^{4} / 4}+b^{2} / 2\right)^{1 / 2}$. The convexity condition (10.7) is valid for all $x \in[0,1)$ and all $p \geq 0$ if and only if $10+2|b| \geq b^{4}$.

Proof. We substitute $\xi^{2}=t$ and obtain that the function $\varrho(\lambda)$ can be rewritten into parameter representation

$$
\lambda(t)=(1+t)^{-p} \mathrm{e}^{-x g(t)}, \quad \varrho(t)=(1+t)^{-p} \mathrm{e}^{-g(t)}, \quad 0 \leq t<\infty .
$$

We introduce the both functions

$$
r(t)=(1+t)^{-p} \mathrm{e}^{(1-x) g(t)}, \quad h(t)=p /(1+t)+\dot{g}(t),
$$

observe that $\lambda(t)=\varrho(t) r(t)$ and $\dot{\varrho}(t)=-\varrho(t) h(t)$, proceed as in the proof of Proposition 3.1 and obtain that $\varrho^{\prime \prime} \geq 0$ is equivalent to $h \ddot{r} / \dot{r} \leq h^{2}+\dot{h}$ for all $t \in[0, \infty)$, which is equivalent to $(10.7)$ and holds true for all $x \in[0,1)$ and all $p \geq 0$ if and only if

$$
\dot{g}-\ddot{g} / \dot{g} \geq 1 /(1+t) \quad \text { for all } t \in[0, \infty) .
$$

This convexity assumption is valid if and only if $10+2|b| \geq b^{4}$.

Step 4 (Deriving the conditional stability estimate): Due to the preparatory steps $1-3$ we are now able to apply the general Theorems 2.1 and 2.3 and obtain

Theorem 10.2. Let (10.7) be valid, then on the set $M$ given by (10.2) we have for $x \in(0,1)$ an improved Hölder type conditional stability estimate and for $x=0$ some logarithmic type conditional stability estimate $\|f\| \leq \beta(\|g\|)$ with

$$
\beta(\delta)=E\left(1+\xi_{0}^{2}\right)^{-p / 2} \mathrm{e}^{-x \operatorname{Re} \theta\left(\xi_{0}\right)}=E^{1-x} \delta^{x}\left(1+\xi_{0}^{2}\right)^{-p(1-x) / 2}
$$

where $\xi_{0}$ is the unique positive solution of the equation $\left(1+\xi^{2}\right)^{-p / 2} \mathrm{e}^{-\operatorname{Re} \theta(\xi)}=\delta / E$. For $\delta \rightarrow 0$ there holds the asymptotic representation

$$
\beta(\delta)=E^{1-x} \delta^{x}\left(\sqrt{2} \ln \delta^{-1}\right)^{-2 p(1-x)}(1+o(1)) .
$$

If the convexity condition (10.7) is violated, then (10.8) and (10.9) hold true with an additional factor $\sqrt{2}$ on the right hand side. 
Proof (sketch). From (10.6) we obtain that the inverse $\varrho^{-1}$ is implicitly given by

$$
\varrho^{-1}\left(\left(1+\xi^{2}\right)^{-p} \mathrm{e}^{-2 \operatorname{Re} \theta}\right)=\left(1+\xi^{2}\right)^{-p} \mathrm{e}^{-2 x \operatorname{Re} \theta}, \quad 0 \leq \xi<\infty .
$$

Hence, (10.8) follows. From $\left(1+\xi_{0}^{2}\right)^{-p / 2} \mathrm{e}^{-\operatorname{Re} \theta\left(\xi_{0}\right)}=\delta / E$ we have

$$
\xi_{0}=2\left(\ln \delta^{-1}\right)^{2}(1+o(1)) \text { for } \delta \rightarrow 0 .
$$

From this asymptotic representation and (10.8) we obtain (10.9).

The asymptotic representation (10.9) tells us that the convection term $b u_{x}$ in (10.1) has no influence on the conditional stability function $\beta$ for $\delta \rightarrow 0$.

\section{Fractional sideways heat conduction}

This problem is taken from [81]. In the special case $\alpha=1$ this problem has been considered, e.g., in $[20,21,22,51,60,70]$. Time fractional diffusion equations are used when attempting to describe transport processes with long memory where the rate of diffusion is inconsistent with the classical Brownian motion model.

System equation formulation of the problem: Determine, for any fixed $x \in[0,1)$, the function $f(t):=u(x, t)$ from the data function $g(t):=u(1, t)$ where $u(x, t)$ obeys the following time fractional sideways heat conduction problem

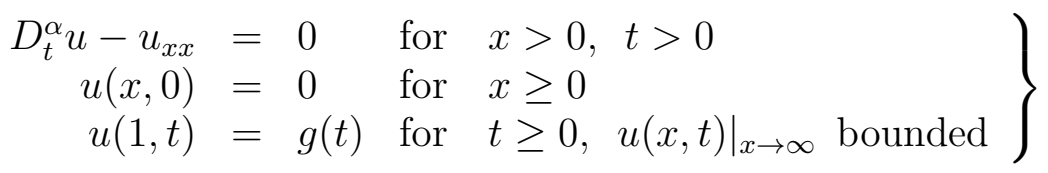

and $D_{t}^{\alpha} u$ is the Caputo fractional derivative of order $\alpha \leq 1$ with respect to $t$, for which there holds $\mathcal{F}\left(D_{t}^{\alpha} u\right)=(i \xi)^{\alpha} \widehat{u}(x, \xi)$. We are in particular interested in the influence of the parameter $\alpha$ on the conditional stability of the above problem.

Smoothness assumption: Let $X=L^{2}(\mathbb{R})$ with norm $\|\cdot\|$. Since we are working with Fourier transform with respect to the variable $t$, we extend the domain of the appearing functions with respect to $t$ by defining them to be zero for $t<0$. For studying conditional stability, we assume for some $E>0$ and $p \geq 0$ in case $x \in(0,1)$ and $p>0$ in case $x=0$ the solution smoothness $\|u(0, t)\|_{p} \leq E$ where $\|\cdot\|_{p}$ is the norm in the Sobolev space $H^{p}(\mathbb{R})$ of order $p \geq 0$, see (5.3). That is, we assume the solution smoothness

$$
u(x, \cdot) \in M=\left\{u(x, \cdot) \in X \mid u \text { obeys }(11.1),\|u(0, \cdot)\|_{p} \leq E\right\}
$$

and ask for a stability estimate $\|f\| \leq \beta(\|g\|)$, or equivalently, $\|\widehat{f}\| \leq \beta(\|\widehat{g}\|)$.

Step 1 (Operator equation formulation of the problem): By using Fourier transform we obtain in the frequency space

$$
\widehat{u}(x, \xi)=e^{-\theta(\xi) x} \widehat{u}(0, \xi) \quad \text { with } \quad \theta(\xi)=|\xi|^{\alpha / 2} \mathrm{e}^{\mathrm{i} \operatorname{sgn}(\xi) \alpha \pi / 4} .
$$

Consequently, $\widehat{u}(0, \xi)=e^{\theta(\xi) x} \widehat{u}(x, \xi)=e^{\theta(\xi)} \widehat{u}(1, \xi)$. We conclude that in the frequency space we have the operator equation $\widehat{A}(x) \widehat{u}(x, \xi)=\widehat{u}(1, \xi)$, or equivalently,

$$
\widehat{A} \hat{f}=\widehat{g} \quad \text { with } \quad \widehat{A} \widehat{f}=\mathrm{e}^{-\theta(\xi)(1-x)} \widehat{f}(\xi) .
$$


Step 2 (Deriving the index function $\varphi$ ): Now we ask the question if the set $M$ is equivalent to some general source set

$$
M_{\varphi, E}=\left\{u(x, \cdot) \in X \mid u(x, \cdot)=\left[\varphi\left(A^{*} A\right)\right]^{1 / 2} v,\|v\| \leq E\right\}
$$

with some index function $\varphi=\varphi(\lambda)$. Some formal computations show that both sets $(11.2)$ and $(11.3)$ are equal if $\varphi:(0,1] \rightarrow(0,1]$ is implicitly given by

$$
\varphi\left(\mathrm{e}^{-2 r(1-x) \cos (\alpha \pi / 4)}\right)=\left(1+r^{4 / \alpha}\right)^{-p} \mathrm{e}^{-2 r x \cos (\alpha \pi / 4)}, \quad 0 \leq r<\infty .
$$

It can be shown that the function $\varphi$ defined by (11.4) is an index function.

Step 3 (Deriving the function $\varrho$ and verifying its convexity): From (11.4) and $\varrho(\varphi(\lambda))=\lambda \varphi(\lambda)$ we obtain that the function $\varrho:(0,1] \rightarrow(0,1]$ is given by

$$
\varrho\left(\left(1+r^{4 / \alpha}\right)^{-p} \mathrm{e}^{-2 r x \cos (\alpha \pi / 4)}\right)=\left(1+r^{4 / \alpha}\right)^{-p} \mathrm{e}^{-2 r \cos (\alpha \pi / 4)}, \quad 0 \leq r<\infty .
$$

We substitute in (11.5) $2 r \cos (\alpha \pi / 4)=\xi$ and see that $\varrho$ defined by (11.5) has the special form (3.11) with constants

$$
\left.\begin{array}{ll}
c_{1}=(2 \cos (\alpha \pi / 4))^{4 p / \alpha}, & c_{2}=(2 \cos (\alpha \pi / 4))^{4 / \alpha} \\
c_{3}=4 / \alpha, \quad \xi_{0}=0, & z=x
\end{array}\right\} .
$$

From Corollary 3.6 we obtain

Proposition 11.1. The function $\varrho(\lambda)$, defined in parameter representation by (3.11) with constants (11.6), is convex for all $(x, p) \in(0,1] \times[0, \infty)$.

Proof. From Corollary 3.6 we have that $\varrho(\lambda)$ is convex for all $(x, p) \in(0,1] \times[0, \infty)$ if and only if $f_{1}(\alpha) \leq f_{2}(\alpha), \alpha \in(0,1]$, where

$$
f_{1}(\alpha)=\sup _{\xi \in[0, \infty)} \frac{\xi^{4 / \alpha}(1-\xi)}{\xi+4 / \alpha-1}, \quad f_{2}(\alpha)=(2 \cos (\alpha \pi / 4))^{4 / \alpha} .
$$

The function $f_{1}$ is monotonically increasing with $\lim _{\alpha \rightarrow 0} f_{1}(\alpha)=0$ and $f_{1}(1) \approx$ 0.02158. The function $f_{2}(\alpha)$ is monotonically decreasing with $\lim _{\alpha \rightarrow 0} f_{2}(\alpha)=\infty$ and $f_{2}(1)=4$. Hence, $f_{1}(\alpha) \leq f_{2}(\alpha)$ is valid for all $\alpha \in(0,1]$.

Step 4 (Deriving the conditional stability estimate): Due to the preparatory steps $1-3$ we are now able to apply the general Theorem 2.1 and obtain

Theorem 11.2. On the set $M$ given by (11.2) we have for $x \in(0,1)$ an improved Hölder type conditional stability estimate and for $x=0$ some logarithmic type conditional stability estimate $\|f\| \leq \beta(\|g\|)$ with

$$
\beta(\delta)=E\left(1+r_{0}^{4 / \alpha}\right)^{-p / 2} \mathrm{e}^{-r_{0} x \cos (\alpha \pi / 4)}=E^{1-x} \delta^{x}\left(1+r_{0}^{4 / \alpha}\right)^{-p(1-x) / 2}
$$

where $r_{0}$ is the unique solution of the equation $\left(1+r^{4 / \alpha}\right)^{-p / 2} \mathrm{e}^{-r \cos (\alpha \pi / 4)}=\delta / E$. For $\delta \rightarrow 0$ there holds the asymptotic representation

$$
\beta(\delta)=E^{1-x} \delta^{x}\left(\frac{1}{\cos (\alpha \pi / 4)} \ln \frac{1}{\delta}\right)^{-2 p(1-x) / \alpha}(1+o(1)) .
$$


Proof (sketch). From (11.5) we obtain that the inverse $\varrho^{-1}$ is given by

$$
\varrho^{-1}\left(\left(1+r^{4 / \alpha}\right)^{-p} \mathrm{e}^{-2 r \cos (\alpha \pi / 4)}\right)=\left(1+r^{4 / \alpha}\right)^{-p} \mathrm{e}^{-2 r x \cos (\alpha \pi / 4)}, \quad 0 \leq r<\infty .
$$

Hence, (11.7) follows. From $\left(1+r_{0}^{4 / \alpha}\right)^{-p / 2} \mathrm{e}^{-r_{0} \cos (\alpha \pi / 4)}=\delta / E$ we have

$$
r_{0}=\left(\frac{1}{\cos (\alpha \pi / 4)} \ln \frac{1}{\delta}\right)(1+o(1)) \quad \text { for } \quad \delta \rightarrow 0 .
$$

From this asymptotic representation and (11.7) we obtain (11.8).

\section{Identification of heat sources}

Problems of this type have been considered, e. g., in [10, 14, 76, 77, 78, 79]. They arise in different engineering problems. For example, an accurate estimation of the pollution source is crucial for environmental protection in cities with high population, see [77].

System equation formulation of the problem: Determine the source function $f$ from the data function $g$ where $f$ and $g$ obey the heat equation problem

$$
\left.\begin{array}{rlrl}
u_{t}-u_{x x} & =f(x) & & \text { for } 0<x<1,0<t \leq 1 \\
u(x, 0) & =0 & & \text { for } 0 \leq x \leq 1 \\
u_{x}(0, t) & =u_{x}(1, t)=0 & & \text { for } 0 \leq t \leq 1 \\
u(x, 1) & =g(x) & & \text { for } 0 \leq x \leq 1
\end{array}\right\}
$$

Smoothness assumption: Let $X=L^{2}(0,1)$ with norm $\|\cdot\|$. For studying conditional stability, we assume the solution smoothness $f \in M$ with

$$
M=\left\{f \in X \mid\|f\|_{p} \leq E\right\}
$$

where $\|f\|_{p}$ is the norm in the Sobolev space $H^{p}(0,1)$ of order $p>0$, that is,

$$
\|f\|_{p}:=\left(\sum_{n=1}^{\infty}\left(1+n^{2}\right)^{p}\left|\left(f, u_{n}\right)\right|^{2}\right)^{1 / 2} \quad \text { with } \quad u_{n}=\sqrt{2} \cos n \pi x,
$$

and ask for a stability estimate $\|f\| \leq \beta(\|g\|)$.

Step 1 (Operator equation formulation of the problem): By separation of variables one obtains that the function

$$
u(x, t)=\sum_{n=1}^{\infty} \frac{\left(1-e^{-n^{2} \pi^{2} t}\right)}{n^{2} \pi^{2}}\left(f, u_{n}\right) u_{n}
$$

obeys the differential equation along with the initial condition and boundary conditions of (12.1). From this representation we conclude that the operator equation Af $=g, A \in \mathcal{L}(X, X), X=L^{2}(0,1)$, is given by

$$
A f=\sum_{n=1}^{\infty} \sigma_{n}\left(f, u_{n}\right) u_{n}=g \quad \text { with } \quad \sigma_{n}=\frac{1-e^{-n^{2} \pi^{2}}}{n^{2} \pi^{2}} .
$$

Hence, $\sigma_{n}$ are the eigenvalues and $u_{n}$ the corresponding eigenelements of the compact operator $A$. 
Step 2 (Deriving the index function $\varphi$ ): Now we ask the question if the set $M$ is equivalent to some general source set

$$
M_{\varphi, E}=\left\{f \in X \mid f=\left[\varphi\left(A^{*} A\right)\right]^{1 / 2} v,\|v\| \leq E\right\}
$$

with some index function $\varphi$. Some formal computations show that both sets $M$ and $M_{\varphi, E}$ are equal if $\varphi:\left(0,\left(1-\mathrm{e}^{-\pi^{2}}\right)^{2} / \pi^{4}\right] \rightarrow\left(0,2^{-p}\right]$ is implicitly given by

$$
\varphi\left(\left(\frac{1-\mathrm{e}^{-l \pi^{2}}}{l \pi^{2}}\right)^{2}\right)=(1+l)^{-p}, \quad 1 \leq l<\infty .
$$

For showing that $\varphi$ is an index function we rewrite (12.3) into parameter representation $\lambda(l)=\left(\frac{1-\mathrm{e}^{-l \pi^{2}}}{l \pi^{2}}\right)^{2}, \varphi(l)=(1+l)^{-p}, 1 \leq l<\infty$, verify that $\dot{\lambda}(l)<0$ and $\dot{\varphi}(l)<0$ and obtain that $\varphi^{\prime}(\lambda)>0$. Since in addition $\lim _{l \rightarrow \infty} \varphi(l)=0$ we conclude that $\varphi$ is an index function.

Step 3 (Deriving the function $\varrho$ and verifying its convexity): From (12.3) and the representation $\varrho(\varphi(\lambda))=\lambda \varphi(\lambda)$ we obtain that $\varrho:\left(0,2^{-p}\right] \rightarrow\left(0,2^{-p}\left(1-\mathrm{e}^{-\pi^{2}}\right)^{2} / \pi^{4}\right]$ is given by

$$
\varrho\left((1+l)^{-p}\right)=(1+l)^{-p}\left(\frac{1-\mathrm{e}^{-l \pi^{2}}}{l \pi^{2}}\right)^{2}, \quad 1 \leq l<\infty .
$$

Following proposition verifies the convexity of the function $\varrho$.

Proposition 12.1. The function $\varrho$ from (12.4) is convex for all $p>0$.

Proof (sketch). We rewrite (12.4) into parameter representation

$$
\lambda(l)=(1+l)^{-p}, \quad \varrho(l)=(1+l)^{-p}\left(\frac{1-\mathrm{e}^{-l \pi^{2}}}{l \pi^{2}}\right)^{2}, \quad 1 \leq l<\infty
$$

and obtain from $\varrho^{\prime \prime}=(\ddot{\varrho} \dot{\lambda}-\dot{\varrho} \ddot{\lambda}) / \dot{\lambda}^{3}$ and $\dot{\lambda}(l)<0$ that $\varrho^{\prime \prime}>0$ is equivalent to $\ddot{\varrho} \dot{\lambda}<\ddot{\varrho}$. From $\varrho(l):=\lambda(l) r(l)$ with $r(l):=\left(\frac{1-\mathrm{e}^{-l \pi^{2}}}{l \pi^{2}}\right)^{2}$ and $\dot{r}(l)<0$ we conclude that $\varrho^{\prime \prime}>0$ is equivalent to $\lambda \ddot{\lambda}-2 \dot{\lambda}^{2}<\lambda \dot{\lambda} \ddot{r} / \dot{r}$, which is equivalent to $(1+l) \ddot{r} / \dot{r}+1<p$ and holds true for all $p>0$ and all $l \in[1, \infty)$.

Step 4 (Deriving the conditional stability estimate): Due to the preparatory steps $1-3$ we are now able to apply the general Theorem 2.1 and obtain

Theorem 12.2. On the set $M$ there holds the Hölder type conditional stability estimate $\|f\| \leq \beta(\|g\|)$ with

$$
\beta(\delta)=E\left(1+t_{0}\right)^{-p / 2}=\pi^{\frac{2 p}{p+2}} E^{\frac{2}{p+2}} \delta^{\frac{p}{p+2}}(1+o(1)) \quad \text { for } \quad \delta \rightarrow 0
$$

where $t_{0}$ is the unique solution of the equation $(1+t)^{-p / 2}\left(\frac{1-\mathrm{e}^{-t \pi^{2}}}{t \pi^{2}}\right)=\delta / E$. 
Proof (sketch). From (12.4) we obtain that the inverse $\varrho^{-1}$ is implicitly given by

$$
\varrho^{-1}\left((1+t)^{-p}\left(\frac{1-\mathrm{e}^{-t \pi^{2}}}{t \pi^{2}}\right)^{2}\right)=(1+t)^{-p}, \quad 1 \leq t<\infty .
$$

Hence, the first representation of (12.5) follows. From (12.6) we obtain that the inverse $\varrho^{-1}$ possesses the asymptotic (explicit) representation

$$
\rho^{-1}(\lambda)=\left(\pi^{4} \lambda\right)^{\frac{p}{p+2}}(1+o(1)) \quad \text { for } \lambda \rightarrow 0 .
$$

From (12.7) and (2.2) we obtain the second representation of (12.5).

\section{Analytic continuation}

Problems of this type have been considered, e. g., in $[13,17,18,80]$. They arise in different important applications such as in medical imaging, see [15].

Problem formulation: Let $h(z)=h(x+i y)=u(x, y)+i v(x, y)$ be an analytic function on $\Omega=\left\{z \in \mathbb{C} \mid x \in \mathbb{R}, 0 \leq y \leq y_{0}\right\}$. Determine, for any fixed $y \in\left(0, y_{0}\right]$, the function $f(x):=h(x+i y)$ from the data $g(x):=\left.h(z)\right|_{y=0} \in X=L^{2}(\mathbb{R})$.

Smoothness assumption: Let $X=L^{2}(\mathbb{R})$ with norm $\|\cdot\|$. For studying conditional stability, we assume for some $E>0$ and $p \geq 0$ in case $y \in\left(0, y_{0}\right)$ and $p>0$ in case $y=y_{0}$ the solution smoothness

$$
f \in M=\left\{f \in X \mid h(z) \text { is analytic on } \Omega,\left\|h\left(\cdot+i y_{0}\right)\right\|_{p} \leq E\right\}
$$

where $\|\cdot\|_{p}$ is the norm in the Sobolev space $H^{p}(\mathbb{R})$ of order $p \geq 0$, see (5.3), and ask for a stability estimate $\|f\| \leq \beta(\|g\|)$, or equivalently, $\|\widehat{f}\| \leq \beta(\|\widehat{g}\|)$.

Step 1 (Operator equation formulation of the problem): From [17, 18] we have that the operator equation formulation of the above problem is given by

$$
\widehat{A} \widehat{f}=\widehat{g} \quad \text { with } \quad \widehat{A} \widehat{f}:=e^{\xi y} \widehat{f}(\xi)
$$

where, for any fixed $y \in\left(0, y_{0}\right]$, the function $\widehat{f}(\xi)$ is the Fourier transformation of $f(x):=h(x+i y)$ with respect to the variable $x$. From the above representation for the operator $\widehat{A}$ we see that for the spectrum we have $\sigma(\widehat{A})=(0, \infty)$. That is, both $\widehat{A}$ and $\widehat{A}^{-1}$ are unbounded. For deriving conditional stability estimates, we use the decomposition idea as outlined in [56, 69]. We decompose the real axis $\mathbb{R}$ into $\mathbb{R}=I \cup W$ and call $I=(-\infty, 0]$ the ill-posed part and $W=[0, \infty)$ the well-posed part. Next, we decompose the space $X$ into the direct sum

$$
X=X_{1} \oplus X_{2} \quad \text { with } \quad X_{1}=L^{2}(I) \quad \text { and } \quad X_{2}=L^{2}(W) .
$$

We introduce $P_{1}, P_{2}$ as the orthoprojections onto $X_{1}$ and $X_{2}$, respectively, and decompose (for fixed $\left.y \in\left(0, y_{0}\right]\right)$ the element $\widehat{f}(\xi)=\mathcal{F}(h(x+i y))$ into the sum

$$
\widehat{f}(\xi)=\widehat{f_{1}}(\xi)+\widehat{f}_{2}(\xi) \quad \text { with } \quad \widehat{f_{1}}(\xi)=P_{1} \widehat{f}(\xi) \quad \text { and } \quad \widehat{f}_{2}(\xi)=P_{2} \widehat{f}(\xi) .
$$

This decomposition allows to decompose the above operator equation in the frequency domain into two separate problems, one ill-posed problem

$$
\widehat{A}_{1} \widehat{f}_{1}(\xi):=e^{\xi y} \widehat{f}_{1}(\xi)=\widehat{g}_{1}(\xi), \quad \widehat{A}_{1}: X_{1} \rightarrow X_{1}, \widehat{g}_{1}:=P_{1} \widehat{g},
$$


and one well-posed problem

$$
\widehat{A}_{2} \widehat{f}_{2}(\xi):=e^{\xi y} \widehat{f}_{2}(\xi)=\widehat{g}_{2}(\xi), \quad \widehat{A}_{2}: X_{2} \rightarrow X_{2}, \widehat{g}_{2}:=P_{2} \widehat{g} .
$$

The well-posed problem is stable and, since $\left\|\widehat{A}_{2}^{-1}\right\| \leq 1$, we have $\left\|\widehat{f}_{2}\right\| \leq\left\|\widehat{g}_{2}\right\|$. It remains to find a stability estimate $\left\|\widehat{f}_{1}\right\| \leq \beta\left(\left\|\widehat{g}_{1}\right\|\right)$ for the ill-posed part.

Step 2 (Deriving the index function $\varphi$ for the ill-posed part): It can be shown that $h\left(\widehat{x+i y_{0}}\right)=e^{-\xi\left(y_{0}-y\right)} \widehat{f}(\xi)$. Hence, $\left\|h\left(\cdot+i y_{0}\right)\right\|_{p} \leq E$ is equivalent to the smoothness assumption $\left\|e^{-\xi\left(y_{0}-y\right)}\left(1+\xi^{2}\right)^{p / 2} \widehat{f}(\xi)\right\|_{L^{2}(\mathbb{R})} \leq E$. We conclude that

$$
\int_{I} e^{-2 \xi\left(y_{0}-y\right)}\left(1+\xi^{2}\right)^{p}\left|\widehat{f}_{1}(\xi)\right|^{2} \mathrm{~d} \xi \leq E_{1}^{2}
$$

where $I=(-\infty, 0]$ is the ill-posed part, $\widehat{f}_{1}=P_{1} \widehat{f}$ and $E_{1} \leq E$. Now we ask the question if the set of functions $\widehat{f}_{1}(\xi)$ that obey $(13.2)$ is equivalent to

$$
M_{\varphi, E_{1}}=\left\{\widehat{f}_{1}(\xi) \in X_{1}\|\|\left[\varphi\left(\widehat{A}_{1}^{*} \widehat{A}_{1}\right)\right]^{-1 / 2} \widehat{f}_{1}(\xi) \|_{L^{2}(I)} \leq E_{1}\right\}
$$

with some index function $\varphi=\varphi(\lambda)$. Some formal computations show that both sets are equal if $\varphi:(0,1] \rightarrow(0,1]$ is implicitly given by

$$
\varphi\left(\mathrm{e}^{2 y \xi}\right)=\left(1+\xi^{2}\right)^{-p} \mathrm{e}^{2 \xi\left(y_{0}-y\right)}, \quad-\infty<\xi \leq 0 .
$$

It can be shown that the function $\varphi$ defined by (13.4) is an index function.

Step 3 (Deriving the function $\varrho$ for the ill-posed part and verify its convexity): From (13.4) we obtain that the function $\varrho(\lambda):=\lambda \varphi^{-1}(\lambda):(0,1] \rightarrow(0,1]$ is implicitly given by

$$
\varrho\left(\left(1+\xi^{2}\right)^{-p} \mathrm{e}^{2 \xi\left(y_{0}-y\right)}\right)=\left(1+\xi^{2}\right)^{-p} \mathrm{e}^{2 \xi y_{0}}, \quad-\infty<\xi \leq 0 .
$$

We substitute in (13.5) $2 \xi y_{0}=-r$ and then $r=\xi$ and see that $\varrho$ defined by (13.5) has the special form (3.11) with constants

$$
c_{1}=\left(2 y_{0}\right)^{2 p}, \quad c_{2}=\left(2 y_{0}\right)^{2}, \quad c_{3}=2, \quad \xi_{0}=0 \quad \text { and } \quad z=\left(y_{0}-y\right) / y_{0} .
$$

From Proposition 3.5 and Corollary 3.6 we obtain

Proposition 13.1. The function $\varrho(\lambda)$, defined in parameter representation by (3.11) with constants (13.6), is convex if and only if (3.12) holds true with constants $\left(c_{2}, c_{3}, \xi_{0}, z\right)$ given by (13.6). This convexity condition is valid for all $(y, p) \in\left(0, y_{0}\right] \times[0, \infty)$ if and only if $y_{0} \geq \frac{1}{2} \sqrt{\sup _{\xi \in[0, \infty)}\left(\xi^{2}-\xi^{3}\right) /(1+\xi)} \approx 0.1514$.

Step 4 (Deriving the conditional stability estimate): Due to the preparatory steps $1-3$ we are now able to apply the general Theorems 2.1 and 2.3 and obtain

Theorem 13.2. Let $\varrho$ defined by (13.5) be convex. Then, on the set (13.2) we have for the ill-posed part for any fixed $y \in\left(0, y_{0}\right)$ an improved Hölder type conditional stability estimate and for $y=y_{0}$ some logarithmic type conditional stability estimate $\left\|f_{1}\right\| \leq \beta\left(\left\|g_{1}\right\|\right)$ with

$$
\beta(\delta)=E_{1}\left(1+\xi_{0}^{2}\right)^{-p / 2} \mathrm{e}^{\xi_{0}\left(y_{0}-y\right)}=E_{1}^{y / y_{0}} \delta^{1-y / y_{0}}\left(1+\xi_{0}^{2}\right)^{-p y /\left(2 y_{0}\right)}
$$


where $\xi_{0}$ is the unique negative solution of the equation $\left(1+\xi^{2}\right)^{-p / 2} \mathrm{e}^{\xi y_{0}}=\delta / E_{1}$. For $\delta \rightarrow 0$ there holds the asymptotic representation

$$
\beta(\delta)=E_{1}^{y / y_{0}} \delta^{1-y / y_{0}}\left(\frac{1}{y_{0}} \ln \frac{1}{\delta}\right)^{-p y / y_{0}}(1+o(1)) .
$$

If $\varrho$ is not convex, then (13.7) and (13.8) hold true with an additional factor $\sqrt{2}$ on the right hand side.

Proof (sketch). From (13.5) we obtain that the inverse $\varrho^{-1}$ is implicitly given by

$$
\varrho^{-1}\left(\left(1+\xi^{2}\right)^{-p} \mathrm{e}^{2 \xi y_{0}}\right)=\left(1+\xi^{2}\right)^{-p} \mathrm{e}^{2 \xi\left(y_{0}-y\right)}, \quad-\infty<\xi \leq 0 .
$$

Hence, (13.7) follows. From $\left(1+\xi_{0}^{2}\right)^{-p / 2} \mathrm{e}^{\xi_{0} y_{0}}=\delta / E_{1}$ we have

$$
\xi_{0}=-\left(\frac{1}{y_{0}} \ln \frac{1}{\delta}\right)(1+o(1)) \quad \text { for } \quad \delta \rightarrow 0 .
$$

From this asymptotic representation and (13.7) we obtain (13.8).

Remark 13.3. From the above two stability estimates (13.8) for the ill-posed part and $\left\|f_{2}\right\| \leq\left\|g_{2}\right\|$ for the well-posed part we obtain due to the Pythagoras Theorem that on the set $M$ defined by (13.1) we have $\|f\| \leq \beta(\|g\|)$ with

$$
\beta(\delta)=E^{y / y_{0}} \delta^{1-y / y_{0}}\left(\frac{1}{y_{0}} \ln \frac{1}{\delta}\right)^{-p y / y_{0}}(1+o(1)) \text { for } \delta \rightarrow 0 .
$$

\section{Acknowledgments}

This work was started while the second author held an appointment as Visiting Professor at the University of Applied Sciences Zittau/Görlitz from June until August 2010 and was supported by DFG (Deutsche Forschungsgemeinschaft) under a cooperative bilateral research project grant. Moreover, Uno Hämarik was supported by the Estonian Science Foundation under the research grant 7489, Bernd Hofmann was supported by the Deutsche Forschungsgemeinschaft (DFG) under the research grant HO 1454/8-1 and Yuanyuan Shao was supported by the Saxon State Ministry of Science and Art (SMWK).

\section{References}

1. G. Alessandrini, L. Rondi, E. , Rosset, and S. Vessella, The stability for the Cauchy problem for elliptic equations, Inverse Problems 25 (2009), 123004 (47pp).

2. K. A. Ames and B. Straughan, Non-Standard and Improperly Posed Problems, Academic Press, London, 1997.

3. D. D. Ang, R. Gorenflo, V. K. Le, and D. D. Trong, Moment Theory and some Inverse Problems in Potential Theory and Heat Conduction, Lect. Notes Math. 1792, Springer, Berlin, 2002.

4. W. Arendt and T. Regińska, An ill-posed boundary value problem for the Helmholtz equation on Lipschitz domain, J. Inv. Ill-Posed Problems 17 (2009), 703-711.

5. J. V. Beck, B. Blackwell, and R. C. St. Clair, Inverse Heat Conduction: Ill-Posed Problems, Wiley-Interscience, New York, 1985.

6. P. Bégout and F. Soria, A generalized interpolation inequality and its application to the stabilization of damped equations, J. Diff. Equ. 240 (2007), 324-356. 
7. A. Böttcher, B. Hofmann, U. Tautenhahn, and M. Yamamoto, Convergence rates for Tikhonov regularization from different kinds of smoothness conditions, Appl. Anal. 85 (2006), 555-578.

8. L. Bourgeois and J. Dardé, About stability and regularization of ill-posed elliptic Cauchy problems: the case of Lipschitz domains, Appl. Anal. 89 (2010), 1745-1768.

9. H. Chen, C. L. Fu, and X. L. Feng, An optimal filtering method for the Cauchy problem of the Helmholtz equation, Appl. Math. Lett. 24 (2011), 958-964.

10. W. Chen, L. L. Zhao, and C. L. Fu, Source term identification for an axisymmetric inverse heat conduction problem, Comput. Math. Appl. 59 (2010), 142-148.

11. H. Cheng, C. L. Fu, and X. L. Feng, Determining surface heat flux in the steady state for the Cauchy problem for the Laplace equation, Appl. Math. Comput. 211 (2009), 374-382.

12. J. Cheng and M. Yamamoto, One new strategy for a priori choice of regularizing parameters in Tikhonov's regularization, Inverse Problems 16 (2000), L31-L38.

13. Z. L. Deng, C. L. Fu, X. L. Feng, and Y. X. Zhang, A mollification regularization method for stable analytic continuation, Math. Comput. Simulat. 81 (2011), 1593-1608.

14. F. F. Dou and C. L. Fu, Determining an unknown source in the heat equation by a wavelet dual least squares method, Appl. Math. Lett. 22 (2009), 661-667.

15. C. L. Epstein, Introduction to the Mathematics of Medical Imaging, SIAM, Philadelphia, 2008.

16. X. L. Feng, Z. Qian, and C. L. Fu, Numerical approximation of solution of nonhomogeneous backward heat conduction problem in bounded region, Math. Comput. Simulat. 79 (2008), $177-188$.

17. C. L. Fu, Z. L. Deng, X. L. Feng, and F. F. Dou, A modified Tikhonov regularization for stable analytic continuation, SIAM J. Numer. Anal. 47 (2009), 2982-3000.

18. C. L. Fu, F. F. Dou, X. L. Feng, and Z. Qian, A simple regularization method for stable analytic continuation, Inverse Problems 24 (2008), 065003 (15pp).

19. C. L. Fu, H. F. Li, X. T. Xiong, and P. Fu, Optimal Tikhonov approximation for a sideways parabolic equation, Int. J. Math. Math. Sci. 8 (2005), 1221-1237.

20. C. L. Fu and Z. Qian, Numerical pseudodifferential operator and Fourier regularization, Adv. Comput. Math. 33 (2010), 449-470.

21. C. L. Fu, X. T. Xiong, and P. Fu, Fourier regularization method for solving the surface heat flux from interior observations, Math. Comput. Model. 42 (2005), 489-498.

22. C. L. Fu, X. T. Xiong, H. F. Li, and Y. B. Zhu, Wavelet and spectral regularization methods for a sideways parabolic equation, Appl. Math. Comput. 160 (2005), 881-908.

23. R. Gorenflo and F. Mainardi, Fractional calculus: integral and differential equations of fractional order, Fractals and Fractional Calculus in Continuum Mechanics (New York) (A. Carpinteri and F. Mainardi, eds.), Springer, 1997, pp. 223-276.

24. M. Hegland, An optimal order regularization method which does not use additional smoothness assumptions, SIAM J. Numer. Anal. 5 (1992), 1446-1461.

25. _ Variable Hilbert scales and their interpolation inequalities with application to Tikhonov regularization, Appl. Anal. 59 (1995), 207-223.

26. M. Hegland and B. Hofmann, Errors of regularisation under range inclusions using variable Hilbert scales, Paper published electronically 2010 under arXiv:1005.3883v1[math.NA], http://arxiv.org/abs/1005.3883.

27. B. Hofmann and P. Mathé, Some note on the modulus of continuity for ill-posed problems in Hilbert space, Preprint 2011-7, Preprintreihe der Fakultät für Mathematik der TU Chemnitz, Chemnitz 2011.

28. B. Hofmann and P. Mathé, Analysis of profile functions for general linear regularization methods, SIAM J. Numer. Anal. 45 (2007), 1122-1141.

29. B. Hofmann, P. Mathé, and M. Schieck, Modulus of continuity for conditionally stable illposed problems in Hilbert space, J. Inv. Ill-Posed Problems 16 (2008), 567-585.

30. B. Hofmann and M. Yamamoto, On the interplay of source conditions and variational inequalities for nonlinear ill-posed problems, Appl. Anal. 89 (2010), 1705-1727. 
31. V. Ivanov and T. Korolyuk, Error estimates for solutions of ill-posed problems, USSR Comp. Math. Math. Phys. 9 (1969), 35-49.

32. V. K. Ivanov, V. V. Vasin, and V. P. Tanana, Theory of Linear Ill-Posed Problems and Its Applications, VSP, Utrecht, 2002.

33. S. I. Kabanikhin, Convergence rate estimation of gradient methods via conditional stability of inverse and ill-posed problems, J. Inv. Ill-Posed Problems 13 (2005), 259-264.

34. _ Conditional stability stopping rule for gradient methods applied to inverse and illposed problems, J. Inv. Ill-Posed Problems 14 (2006), 805-812.

35. S. I. Kabanikhin and M. Schieck, Impact of conditional stability: Convergence rates for general linear regularization methods, J. Inv. Ill-Posed Problems 16 (2008), 267-282.

36. A. A. Kilbas, H. M. Srivastava, and J. J. Trujillo, Theory and Applications of Fractional Differential Equations, Elsevier, Amsterdam, 2006.

37. M. V. Klibanov and A. A. Timonov, Carleman Estimates for Coefficient Inverse Problems, VSP, Utrecht, 2004.

38. J. Li, M. Yamamoto, and J. Zou, Conditional stability and numerical reconstruction of initial temperature, Comm. Pure Appl. Anal. 8 (2009), 361-382.

39. Z. P. Li and C. L. Fu, A mollification method for a Cauchy problem for the Laplace equation, Appl. Math. Comput. 217 (2011), 9209-9218.

40. P. Mathé and B. Hofmann, Direct and inverse results in variable Hilbert scales, J. Approx. Theory 154 (2008), 77-89.

41. P. Mathé and S. V. Pereverzev, Geometry of linear ill-posed problems in variable Hilbert scales, Inverse Problems 19 (2003), 789-803.

42. P. Mathé and U. Tautenhahn, Interpolation in variable Hilbert scales with application to inverse problems, Inverse Problems 22 (2006), 2271-2297.

43. A. A. Melkman and C. A. Micchelli, Optimal estimation of linear operators in Hilbert spaces from inaccurate data, SIAM J. Numer. Anal. 16 (1979), 87-105.

44. V. A. Mikhailets and A. A. Murach, Interpolation with a function parameter and refined scale of spaces, Methods Funct. Anal. Topology 14 (2008), 81-100.

45. M. T. Nair, S. V. Pereverzev, and U. Tautenhahn, Regularization in Hilbert scales under general smoothing conditions, Inverse Problems 21 (2005), 1851-1869.

46. M. T. Nair, E. Schock, and U. Tautenhahn, Morozov's discrpancy principle under general source conditions, Z. Anal. Anw. 22 (2003), 199-214.

47. M. T. Nair and U. Tautenhahn, Convergence rates for Lavrentiev-type regularization in Hilbert scales, Comput. Meth. Appl. Math. 8 (2008), 279 - 293.

48. L. E. Payne, Improperly Posed Problems in Partial Differential Equations, SIAM, Philadelphia, 1975.

49. Z. Qian, C. L. Fu, and Z. P. Li, Two regularization methods for a Cauchy problem for the Laplace equation, J. Math. Anal. Appl. 338 (2008), 479-489.

50. Z. Qian, C. L. Fu, and R. Shi, A modified method for a backward heat conduction problem, Appl. Math. Comput. 185 (2007), 564-573.

51. Z. Qian, C. L. Fu, and X. T. Xiong, Semidiscrete central difference method in time for determining surface temperatures, Int. J. Math. Math. Sci. 2005 (2005), 393-400.

52. —_ A modified method for a non-standard inverse heat conduction problem, Appl. Math. and Comput. 180 (2006), 453-468.

53. C. Y. Qiu and C. L. Fu, Wavelets and regularization of the Cauchy problem for the Laplace equation, J. Math. Anal. Appl. 338 (2008), 1440-1447.

54. T. Regińska and K. Regiński, A Cauchy problem for the Helmholtz equation: application to analysis of light propagation in solids, Tech. Report 06-04, Univ. Kiel, 2006.

55. _ Approximate solution of a Cauchy problem for the Helmholtz equation, Inverse Problems 22 (2006), 975-989.

56. T. Regińska and U. Tautenhahn, Conditional stability estimates and regularization with applications to Cauchy problems for the Helmholtz equation, Num. Funct. Anal. and Optimiz. 30 (2009), 1065-1097. 
57. T. Regińska and A. Wakulicz, Wavelet moment method for the Cauchy problem for the Helmholtz equation, J. Comput. Appl. Math. 223 (2009), 218-229.

58. F. Riesz and Sz. Nagy, Functional analysis, Dover, Mineola, 1990.

59. U. Tautenhahn, Optimal stable solution of Cauchy problems for elliptic equations, Z. Anal. Anw. 15 (1996), 961-984.

60. _ Optimal stable approximations for the sideways heat equation, J. Inv. Ill-Posed Problems 5 (1997), 287-307.

61. _ Optimality for linear ill-posed problems under general source conditions, Num. Funct. Anal. and Optimiz. 19 (1998), 377-398.

62. U. Tautenhahn and T. Schröter, On optimal regularization methods for the backward heat equation, Z. Anal. Anw. 15 (1996), 475-493.

63. A. N. Tikhonov, On the stability of inverse problems, C. R. (Doklady) Acad. Sci. URSS (N.S.) 39 (1943), 176-179.

64. A. N. Tikhonov and V. Y. Arsenin, Solution of Ill-Posed Problems, Wiley, New York, 1977.

65. G. M. Vainikko, On the optimality of methods for ill-posed problems, Z. Anal. Anw. 6 (1987), 351-362.

66. G. M. Vainikko and A. Y. Veretennikov, Iteration Procedures in Ill-Posed Problems, Nauka, Moscow, 1986, In Russian.

67. S. Vessela, Quantitative estimates of unique continuation for parabolic equations, determination of unknown time-varying boundaries and optimal stability estimates, Inverse Problems 24 (2008), 023001 (81pp).

68. H. Weyl, Über beschränkte quadratische Formen, deren Differenz vollstetig ist, Rend. Circ. Mat. Palermo 27 (1909), 373-392.

69. X. T. Xiong and C. L. Fu, Two approximate methods of a Cauchy problem for the Helmholtz equation, Comput. Appl. Math. 26 (2007), 285-307.

70. _ A spectral regularization method for solving surface heat flux on a general sideways parabolic, Appl. Math. Comput. 197 (2008), 358-365.

71. X. T. Xiong, C. L. Fu, and H. F. Li, Central difference method of a non-standard inverse heat conduction problem for determining surface heat flux from interior observations, Appl. Math. and Comput. 173 (2006), 1265-1287.

72. X. T. Xiong, C. L. Fu, and Z. Qian, Two numerical methods for solving a backward heat conduction problem, Appl. Math. and Comput. 179 (2006), 370-377.

73. _ On three spectral regularization methods for a backward heat conduction problem, J. Korean Math. Soc. 44 (2007), 1281-1290.

74. X. T. Xiong, C. L. Fu, Z. Qian, and X. Gao, Error estimates of a difference approximation method for a backward heat conduction problem, Int. J. Math. Math. Sci. 2006 (2006), 1-9.

75. M. Yamamoto, Carleman estimates for parabolic equations and applications, Inverse Problems 25 (2009), 123013 (75pp).

76. L. Yan, F. L. Yang, and C. L. Fu, A meshless method for solving an inverse spacewisedependent heat source problem, J. Comput. Physics 228 (2009), 123-136.

77. F. Yang and C. L. Fu, A simplified Tikhonov regularization method for determining the heat source, Appl. Math. Model. 34 (2010), 3286-3299.

78. _ . The method of simplified Tikhonov regularization for dealing with the inverse timedependent heat source problem, Comput. Math. Appl. 60 (2010), 1228-1236.

79. __ Two regularization methods to identify time-dependent heat source through an internal measurement of temperature, Appl. Math. Model. 53 (2011), 793-804.

80. Y. X. Zhang, C. L. Fu, and L. Yan, Approximate inverse method for stable analytic continuation in a strip domain, J. Comput. Appl. Math. 235 (2011), 2979-2992.

81. G. H. Zheng and T. Wei, Spectral regularization method for a Cauchy problem of the timefractional advection-dispersion equation, J. Comput. Appl. Math. 233 (2010), 2631-2640.

82. __ Two regularization methods for solving a Riesz-Feller space-fractional backward diffusion problem, Inverse Problems 26 (2010), 115017 (22pp). 
Ulrich Tautenhahn, University of Applied Sciences Zittau/Görlitz, Faculty of Mathematics and Natural Sciences, P.O.Box 1455, 02755 Zittau, Germany

E-mail address: u.tautenhahn@hs-zigr.de

Uno Hämarik, University of Tartu, Institute of Mathematics, J. Lifvi 2-417, 50409 TARTU, Estonia

E-mail address: Uno.Hamarik@ut.ee

Bernd Hofmann, Chemnitz University of Technology, Faculty of Mathematics, 09107 Chemnitz, Germany

E-mail address: hofmannb@mathematik.tu-chemnitz.de

Yuanyuan Shao, University of Applied Sciences Zittau/Görlitz, Faculty of Mathematics and Natural Sciences, P.O.Box 1455, 02755 Zittau, Germany

E-mail address: yshao@hs-zigr.de and yuanyuan. shao@s2009.tu-chemnitz.de 